This item was submitted to Loughborough's Research Repository by the author.

Items in Figshare are protected by copyright, with all rights reserved, unless otherwise indicated.

\title{
Effect of two-year degradation on mechanical interaction between a bioresorbable scaffold and blood vessel
}

PLEASE CITE THE PUBLISHED VERSION

https://doi.org/10.1016/j.jmbbm.2017.11.031

PUBLISHER

(C) Elsevier

VERSION

AM (Accepted Manuscript)

\section{PUBLISHER STATEMENT}

This work is made available according to the conditions of the Creative Commons Attribution-NonCommercialNoDerivatives 4.0 International (CC BY-NC-ND 4.0) licence. Full details of this licence are available at: https://creativecommons.org/licenses/by-nc-nd/4.0/

\section{LICENCE}

CC BY-NC-ND 4.0

\section{REPOSITORY RECORD}

Qiu, Tianyang, Ran He, C. Abunassar, S. Hossainy, and Liguo Zhao. 2017. "Effect of Two-year Degradation on Mechanical Interaction Between a Bioresorbable Scaffold and Blood Vessel”. Loughborough University. https://hdl.handle.net/2134/27561. 


\title{
Effect of Two-Year Degradation on Mechanical Interaction between a Bioresorbable Scaffold and Blood Vessel
}

\author{
T. Qiu $^{1}$, R. He ${ }^{1}$, C. Abunassar ${ }^{2}$, S. Hossainy ${ }^{2}$, L.G. Zhao ${ }^{1 *}$ \\ ${ }^{1}$ Wolfson School of Mechanical, Electrical and Manufacturing Engineering, Loughborough \\ University, Epinal Way, Loughborough, LE11 3TU, UK \\ ${ }^{2}$ Abbott Vascular, 3200 Lakeside Drive, Santa Clara, CA 95054, USA
}

*Corresponding author, Email: L.Zhao@Lbboro.ac.uk, Tel: 0044-1509-227799

\begin{abstract}
This paper aims to evaluate the mechanical behaviour of a bioresorbable polymeric coronary scaffold using finite element method, focusing on scaffold-artery interaction during degradation and vessel remodelling. A series of nonlinear stress-strain responses was constructed to match the experimental measurement of radial stiffness and strength for polymeric scaffolds over 2year in-vitro degradation times. Degradation process was modelled by incorporating the change of material property as a function of time. Vessel remodelling was realised by changing the size of artery-plaque system manually, according to the clinical data in literature. Over degradation times, stress on the scaffold tended to increase firstly and then decreased gradually, corresponding to the changing yield stress of the scaffold material; whereas the stress on the plaque and arterial layers showed a continuous decrease. In addition, stress reduction was also observed for scaffold, plaque and artery in the simulations with the consideration of vessel remodelling. For the first time, the work offered insights into mechanical interaction between a bioresorbable scaffold and blood vessel during two-year in-vitro degradation, which has significance in assisting with further development of bioresorbable implants for treating cardiovascular diseases.
\end{abstract}


Keywords: Bioresorbable polymeric scaffold; Mechanical Interaction; Degradation; Finite element; Vessel remodelling

\section{Introduction}

Bioresorbable scaffolds, made of Poly (lactic-acid) (PLLA), are to provide temporary mechanical support for arterial lumen and then gradually absorbed in human body due to their degradation properties. The duration in which the polymeric scaffolds provide the mechanical support is called functional time, and the duration for the scaffold to degrade is referred as disappearance time (Lyu and Untereker, 2009). The use of bioresorbable scaffolds can reduce the risk of longterm complications that may arise as a result of undesired biological response to a permanent implant, such as late stent thrombosis and chronic tissue inflammation. Moreover, there is no need to remove the stents for an additional surgical intervention.

Experimental studies of degradation behaviour for a range of PLLA scaffolds have been widely carried out. Gong et al. (2007) investigated the in vitro degradation behaviour (i.e., weight loss, water uptake, molecular weight and morphology) of porous PLLA scaffolds over 39 weeks, and found that the scaffolds suffered degradation at a slow rate due to its highly porous structure. The in vitro degradation study by Liu et al. (2014) showed that PLLA porous scaffolds exhibited a reduction in mechanical properties (compressive modulus and strength), mass and molecular weight during a degradation time of 200 days. Rodrigues et al. (2016) carried out an in vitro degradation study of porous PLA scaffold immersed in phosphate-buffered-saline solution for 8 weeks. The study revealed that there was no significant change for molecular weight, whereas compressive properties (i.e., compressive modulus and stress at yield) of the scaffolds maintained constant during the initial 6 weeks and increased significantly at week 8. Zamiri et al. (2010) conducted the in vitro degradation testing of PLLA and PLLA/PLGA braided scaffolds, and 
their results showed that the mass loss was very limited for PLLA braided scaffolds up to 25 weeks while 64\% mass loss was found for PLLA/PLGA braided scaffolds. Grabow et al. (2007) conducted in vitro degradation study for a PLLA scaffold prototype in a deployed shape, and they found that collapse pressure increased firstly within 12 weeks and then deceased, while the molecular weight showed a gradual and steady reduction during the 24 weeks.

Mechanical properties (i.e., ductility, toughness and strength) of biodegradable polymers change significantly during degradation, due to hydrolytic chain scission at molecule level. It is of importance to understand the mechanical behaviour of bioresorbable polymeric scaffolds during degradation period, especially for simulation and prediction of scaffold performance. Efforts have been made to describe the mechanical behaviour of biodegradable polymers during degradation. Soares et al. (2008) proposed a nonlinear constitutive model for degradable polymer, by introducing a scalar field variable that combined the degradation and mechanical (i.e., shear modulus) parameters. The proposed model was also applied in finite element analysis to investigate the mechanical response of PLLA scaffolds with different designs under constant external pressure (Soares et al., 2010). The results showed that PLLA scaffolds degraded faster at the bends of rings and junction points, with a loss of stiffness and a reduction of recoil. Vieira et al. (2011) tried to combine a hyperelastic model (Neo-Hookean or Mooney-Rivlin) with hydrolytic damage to predict the mechanical behaviour of biodegradable polymers during hydrolytic degradation process, and the numerical results were in good agreement with the tensile test data for PLA/PCL fibres. Luo et al. (2014) developed a thermodynamically consistent model for deformation-induced degradation, which was implemented in finite element framework to analyse the degradation of bioresorbable polymeric scaffolds. The simulated degradation rate and scaffold diameter change showed a good agreement with in vitro study. 
On the other hand, several follow-up studies of clinical trials reported that vessel remodelling was observed following the implantation of bioresorbable scaffolds in humans and animals, by multiple invasive imaging methods such as multi-slice computed tomography (CT), angiography and Intravascular Ultrasound (IVUS). Ormiston et al. (2008) reported that the BVS (bioresorbable vascular scaffold) Absorb showed an angiographic in-scaffold loss of $0.44 \mathrm{~mm}$ and an overall $16.8 \%$ reduction of lumen area at 6-month follow-up after implantation in 30 patients. Serruys et al. (2009) addressed the trial outcomes of Absorb after 2-year follow-up, in which the angiographic in-scaffold late loss was $0.48 \mathrm{~mm}$ and the lumen area increased from 5.19 $\mathrm{mm}^{2}$ (at 6 months) to $5.47 \mathrm{~mm}^{2}$. Similarly, Serruys et al. (2010) evaluated the efficacy of Absorb for treatment of de novo coronary artery stenosis in 45 patients. At 6-month follow-up, the mean lumen area reduced by $3.1 \%$ (from $6.60 \mathrm{~mm}^{2}$ to $6.37 \mathrm{~mm}^{2}$ ) according to IVUS analysis. At 2-year follow-up, the mean lumen area showed an increase (from $6.37 \mathrm{~mm}^{2}$ to $6.85 \mathrm{~mm}^{2}$ ) (Ormiston et al., 2012). Also, Serruys et al. (2011) successfully implanted 57 Absorb scaffolds in 56 patients, and the 12-month follow-up results showed that there was a slight increase for the mean lumen area from $6.31 \mathrm{~mm}^{2}$ (Post-PCI) to $6.33 \mathrm{~mm}^{2}$. Recently, they reported 5-year followup results after Absorb implantation, and they concluded that the mean lumen area tended to increase from 6 months to 1 year and 5 years (Serruys et al., 2016). Moreover, progressive lumen gain was also observed in porcine coronary arteries after Absorb implantation (Lane et al., 2014). The authors addressed that the lumen area retained stable from 1 to 6 months and then showed a progressive increase from 12 to 42 months. Therefore, vessel remodelling occurs in terms of lumen gain after scaffold implantation, and is yet to be considered in the study of scaffold-artery interaction.

So far, most computational work has focused on the development and validation of material constitutive models that aim to predict the load-bearing, stress-strain behaviour of degradable polymer or implant (e.g., Soares et al., 2008; Vieira et al., 2011; Luo et al., 2014) or on the 
simulations of polymer degradation with concurrent drug elution from degradable polymer coatings (e.g., Prabhu and Hossainy, 2006; Chen et al., 2011; Zhu and Braatz, 2014). However, computational studies characterizing both biodegradable implant mechanics and concomitant blood vessel stress is critically required. This can help predict the biological outcome due to progressively changing load-bearing property of a vascular implant considering scaffold degradation process. The objective of this study is to characterize scaffold-artery interaction during scaffold degradation, as well as vessel remodelling, using finite element method. Degradation behaviour was modelled by incorporating a series of nonlinear stress-strain responses constructed based on the experimental measurement of scaffold radial stiffness and strength over 2-years of in-vitro degradation. Vessel remodelling was realised by changing the size of plaque manually, according to the clinical measurements described in literature. Then the variation of stresses in scaffold and artery were presented and discussed.

\section{Calibration of stress-strain curves during in-vitro degradation}

\subsection{Radial strength and stiffness of Absorb scaffold}

The radial strength and stiffness of Absorb scaffold was determined using the MSI RX550 Radial Force Tester. The scaffold itself is essentially made of PLLA, i.e., Poly-l-lactic acid, which is a semi-crystalline thermoplastic polymer and also widely used in medical implants in the forms of rods, plates and pins. The entire procedure can be divided into five steps: (I) sample preparation, (II) sample pre-conditioning, (III) scaffold deployment, (IV) scaffold measurement and (V) radial strength \& stiffness testing. In step I, the catheter-balloon-scaffold system was removed from packaging, and then microscope was used to confirm that the crimped scaffold and balloon were properly aligned. In step II, the delivery system was soaked in water bath at $37^{\circ} \mathrm{C}$ for preconditioning for at least 2 minutes. In step III, scaffold deployment in an expansion block was performed by balloon inflation. The balloon inflation and deflation procedures were completed in the water bath at $37^{\circ} \mathrm{C}$. Afterwards, the expanded scaffold was removed from the water bath, 
and the length was measured. Finally, in step V, the expanded scaffold was inserted into crimper head, and compressed to the nominal diameter. The pressure-diameter graph was obtained from this compression process, from which the radial strength and stiffness were determined. Specifically, as shown in Fig. 2, the black data points depict the increase in pressure measured as the diameter of the scaffold is decreased within the tester. The blue line (modulus) fits the linear portion of pressure-diameter curve, whose slope gives the radial stiffness. The radial strength is determined by the intersection of $0.1 \mathrm{~mm}$ offset line (red line) and the pressure-diameter curve. Using this method, the measured radial strength and radial stiffness values (normalised against the value at day zero) for bioresorbable polymeric scaffold at different in-vitro degradation time points are plotted in Fig. 2. It should be pointed out that all data were obtained for the Absorb scaffolds over a 2-year in-vitro degradation time (720 days), corresponding to virtually complete degradation.

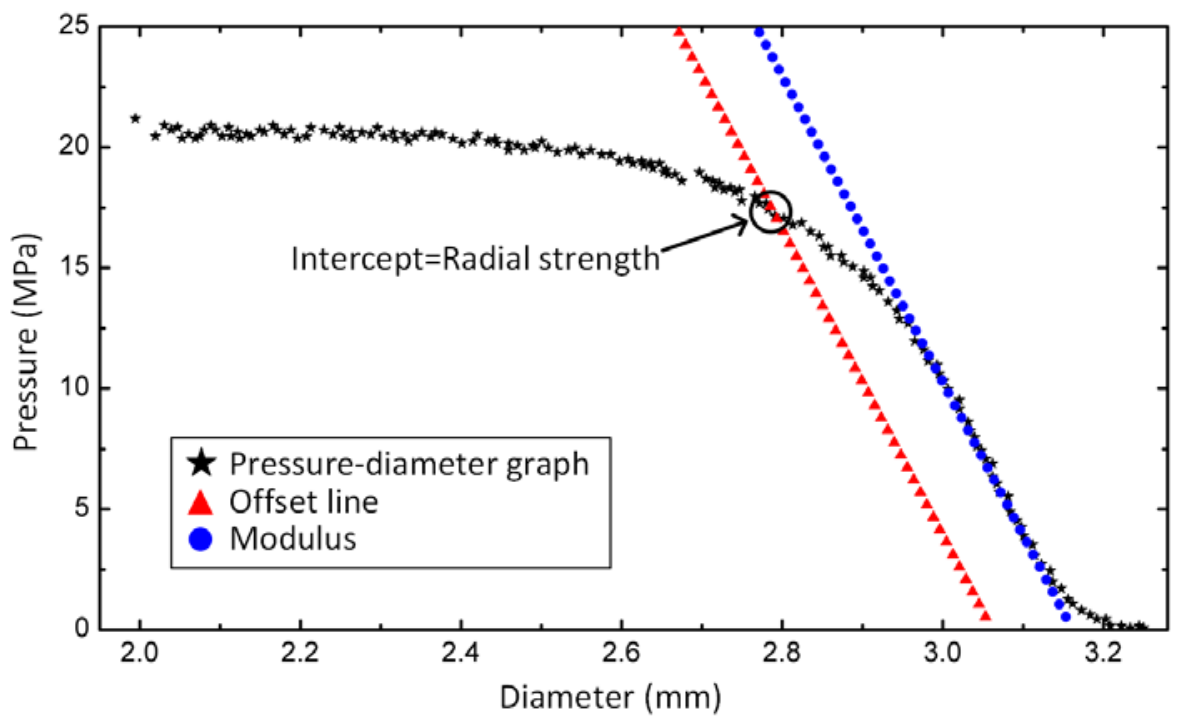

Fig. 1, Pressure-diameter graph obtained from the experimental testing (Absorb scaffold, Abbott Vascular). 


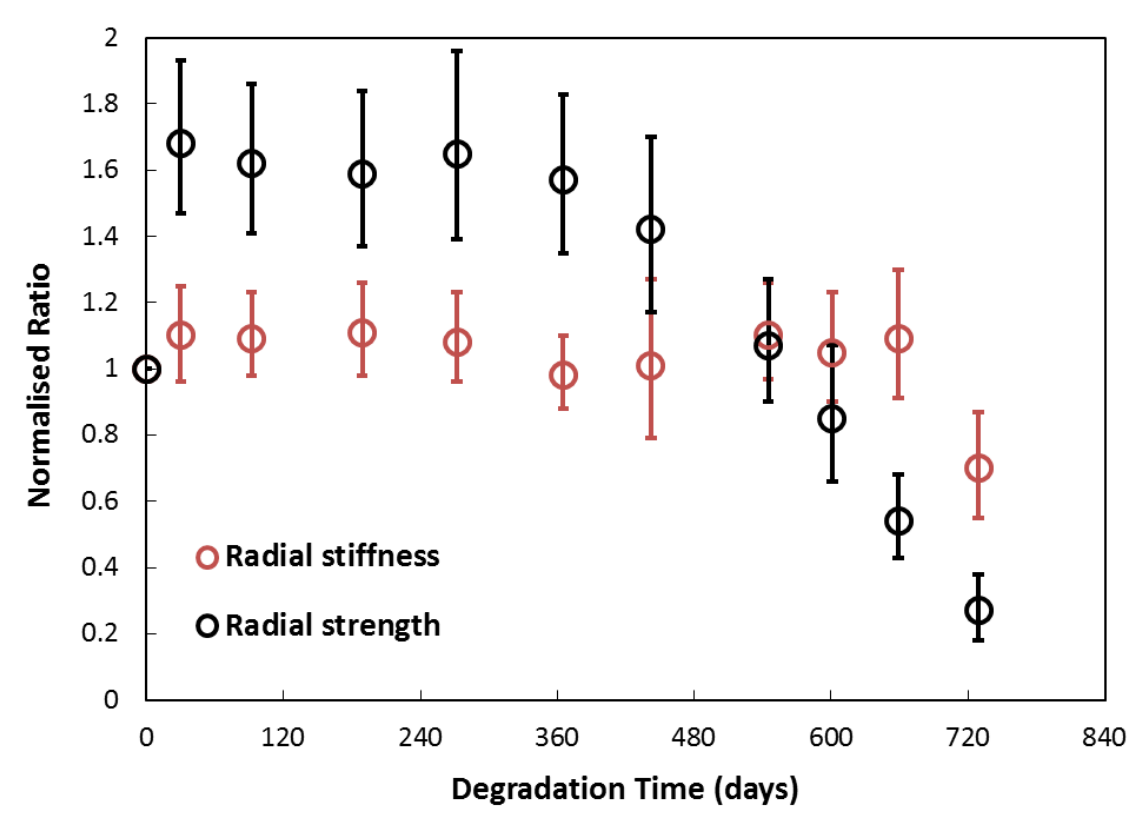

Fig. 2, Radial strength and radial stiffness data for Absorb scaffold (Abbott Vascular).

\subsection{Calibration of stress-strain curves for PLLA during degradation}

The full procedure for calibrating PLLA stress-strain curves is shown in Fig. 3. The stress-strain behaviour was used as model input. Then a simulation was performed to evaluate radial strength and stiffness of scaffold, according to the experimental testing method described above. Specifically, the scaffold was crimped in the first crimping step, in which the displacement loading was applied to 12 rigid plates to compress the original scaffold onto the balloon (as further explained in the following; see Fig. 4b). Afterwards, the crimped scaffold was expanded by balloon inflation, followed by balloon deflation to allow scaffold recoil to occur. The second crimping was subsequently modelled to produce the radial pressure-diameter curve, which was used to determine the radial strength and radial stiffness of the deployed scaffold. This process was performed iteratively, i.e., tuning the stress-strain curves until the simulated radial strength and stiffness match the measured ones. This method is essentially an "inverse" curve-fitting process, for which an iterative adjustment process was used to adjust the stress-strain curve until the simulated radial stiffness and strength matched the test data. 


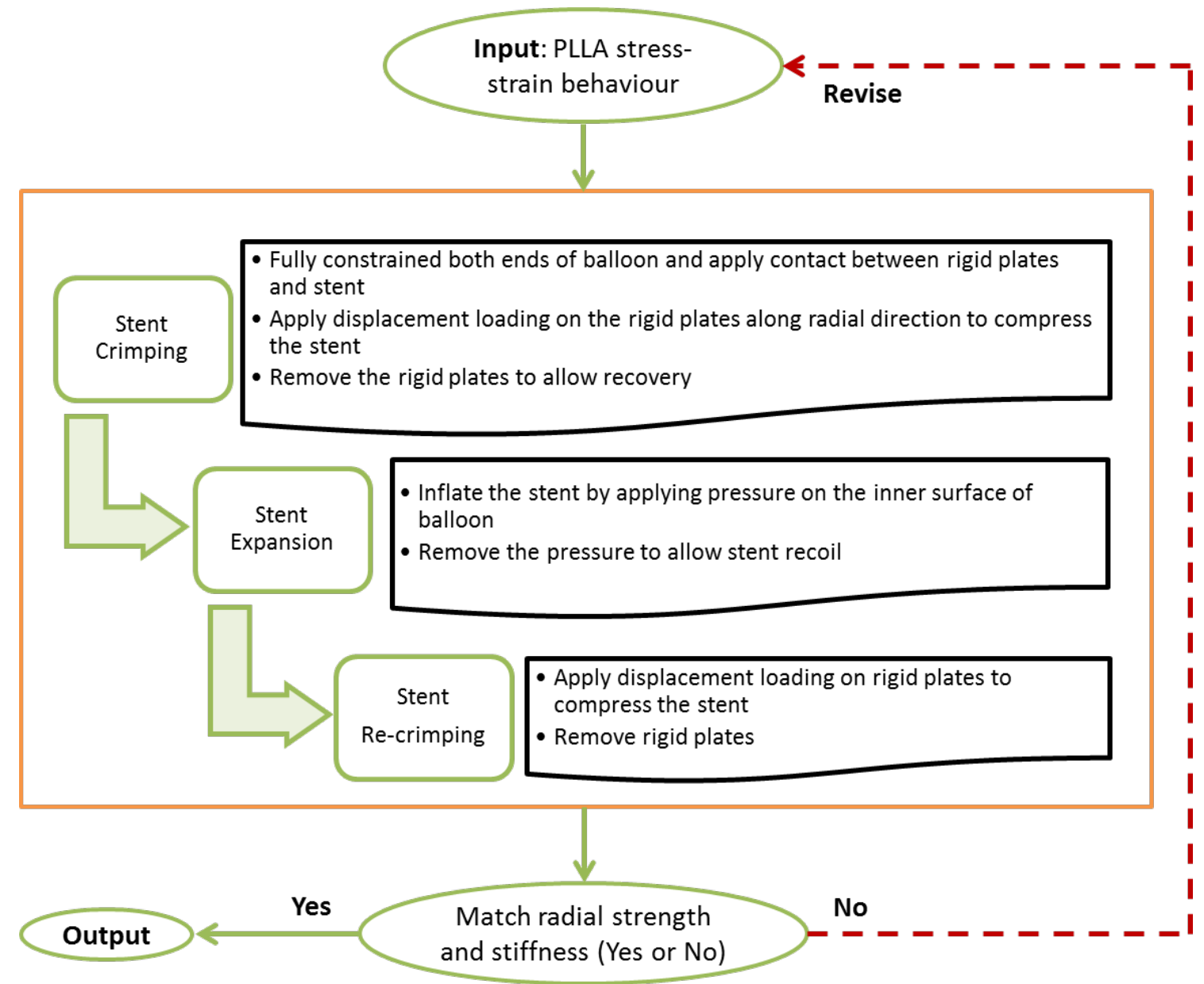

Fig. 3, Flow chart for calibration of PLLA stress-strain curves.

Abaqus 6.14 CAE was used to build the 3D finite element models for Absorb scaffold (Fig. 4a). The scaffold modelled was $12.66 \mathrm{~mm}$ in length and has 13 ring units connected by axial struts (Fig. 4a). Each ring unit has 3 peaks and 3 valleys (Fig. 4a). Dimensions of the Absorb scaffold were measured using OGP flash 200 Microscope. The thickness and width of struts are $150 \mu \mathrm{m}$, while the width at U-bend is $200 \mu \mathrm{m}$ (Fig. 4a). To create the model (Fig. 4a), three steps are required: (i) creating the flat pattern of scaffold, (ii) partitioning and meshing the flat model and (iii) wrapping the meshed flat model to produce tubular model. The flat model is a $2 \mathrm{D}$ version of the scaffold (cut the scaffold open in longitudinal direction), which was then revolved into 3D scaffold using the "wrap-up" tool in ABAQSU CAE. Since this wrapping procedure is only a geometrical operation rather than a mechanical procedure, there is no residual stress generated in 
the scaffold. Hexahedral 8-node elements with reduced integration (C3D8R) were used to mesh the scaffold (Fig. 4a). There are 5 layers of elements through the thickness direction and 6 layers of elements through the width direction (Fig. 4a; based on our mesh sensitivity study). The mechanical property for virgin PLLA was found in Pauck and Reddy (2015) who obtained stressstrain curve through performing uniaxial tensile tests. The stress-strain response was already reported in our recently published papers (Schiavone et al., 2016; 2017) and thus omitted here. For the PLLA material, the density is $1.4 \times 10^{-6}$, Young's Modulus is $2200 \mathrm{MPa}$ and Poisson Ratio is 0.3 . In simulation, plastic behaviour was described by providing the yield stress as a function of plastic strain extracted from the stress-strain curve (Schiavone et al., 2016; 2017). Yielding refers to the transition from linear elastic deformation to nonlinear plastic deformation, and the stress level corresponding to this transition point is called yield stress.

NX 8.5 (Siemens PLM Software, UK) was used to create the tri-folded balloon model (Fig. 4a). Specifically, a series of projected curves were created on four uniformly distributed datum planes, with a largest diameter of $0.4 \mathrm{~mm}$ in fully folded configuration. These cross-sectional sketches were further extruded to produce the $3 \mathrm{D}$ shell by using the sweep tool. Then the $3 \mathrm{D}$ shell was imported into Abaqus CAE to produce the final geometry of tri-folded balloon model (Fig. 4a). The length of tri-folded balloon is $16 \mathrm{~mm}$ for the middle part, and the fully inflated diameter is about $3.2 \mathrm{~mm}$. Four-node quadrilateral membrane element was chosen to mesh the balloon (Fig. 4a). The balloon was defined as an isotropic and linear-elastic material, with Young's Modulus of $900 \mathrm{MPa}$ and Poisson Ratio of 0.3 (Gervaso et al., 2008).

For scaffold crimping, 12 rigid plates (Fig. 4b) were built to squash the scaffold onto the balloon controlled by enforced displacement loading (Schiavone et al., 2016). Both ends of balloon were fully constrained during the simulation and hard contact between scaffold outer surface and rigid plates was specified with a friction coefficient of 0.8 . An additional spring back step was also 
included by releasing the 12 rigid plates after scaffold crimping, which allowed for a recovery of elastic deformation of the crimped scaffold. Images of the simulated scaffold crimping are shown in Fig. 4c.

The radial strength and radial stiffness of the virgin Absorb scaffold were obtained from the simulations using the original PLLA stress-strain curve. The PLLA stress-strain curves at different degradation time points were artificially constructed, and then used as the input to predict radial strength and stiffness results. The goal of this process is to match the simulated scaffold stiffness and strength with the experimental measurements in Fig. 2 by tuning the constructed stress-strain curves. The process is an iteration process based on trial and error as shown in Fig. 3. Here, the radial strength and stiffness, normalised to the virgin state (i.e., zero time point), were used for fitting the stress-strain curves of PLLA at different degradation time points (Fig. 2). 

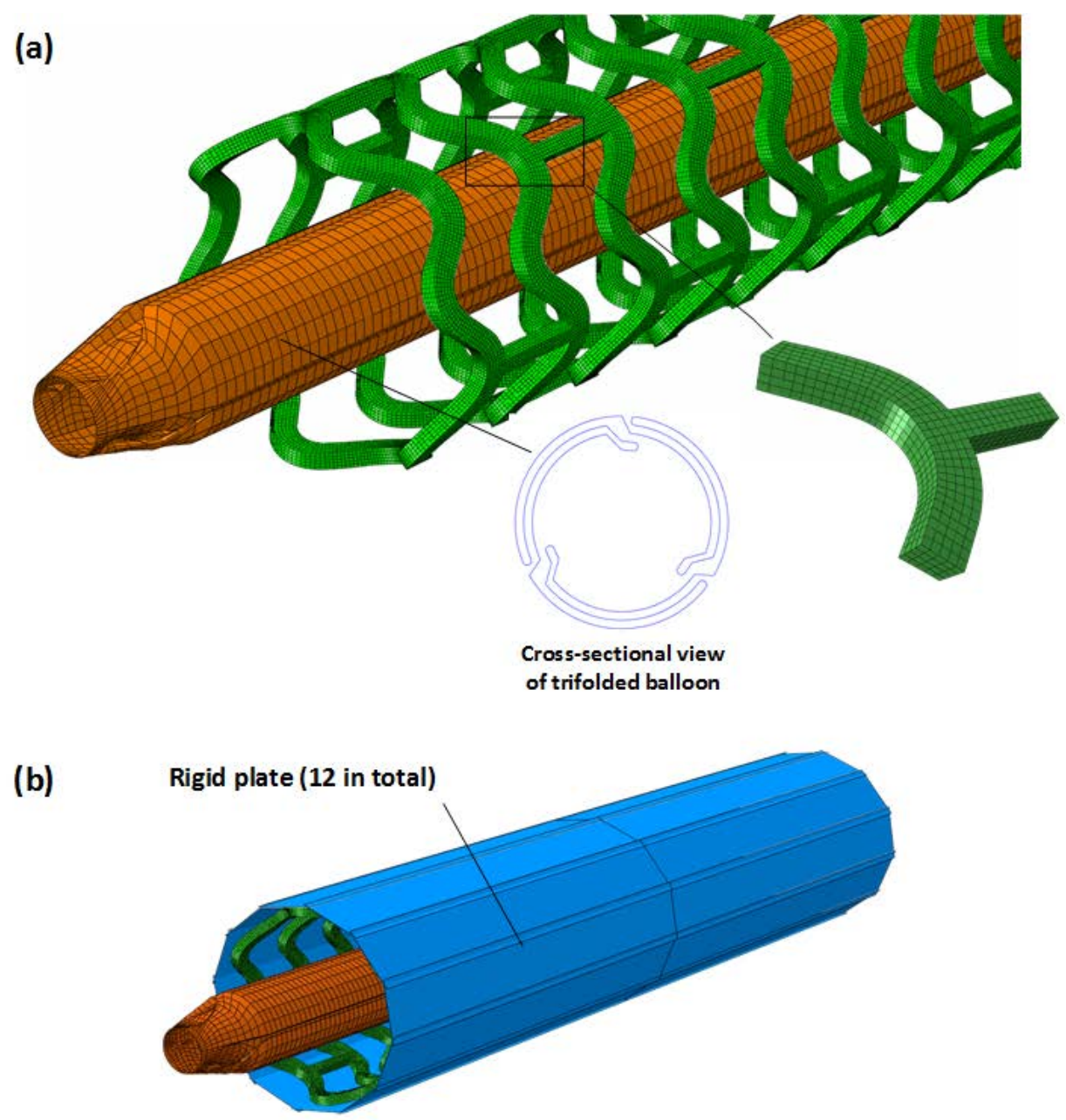

(c)

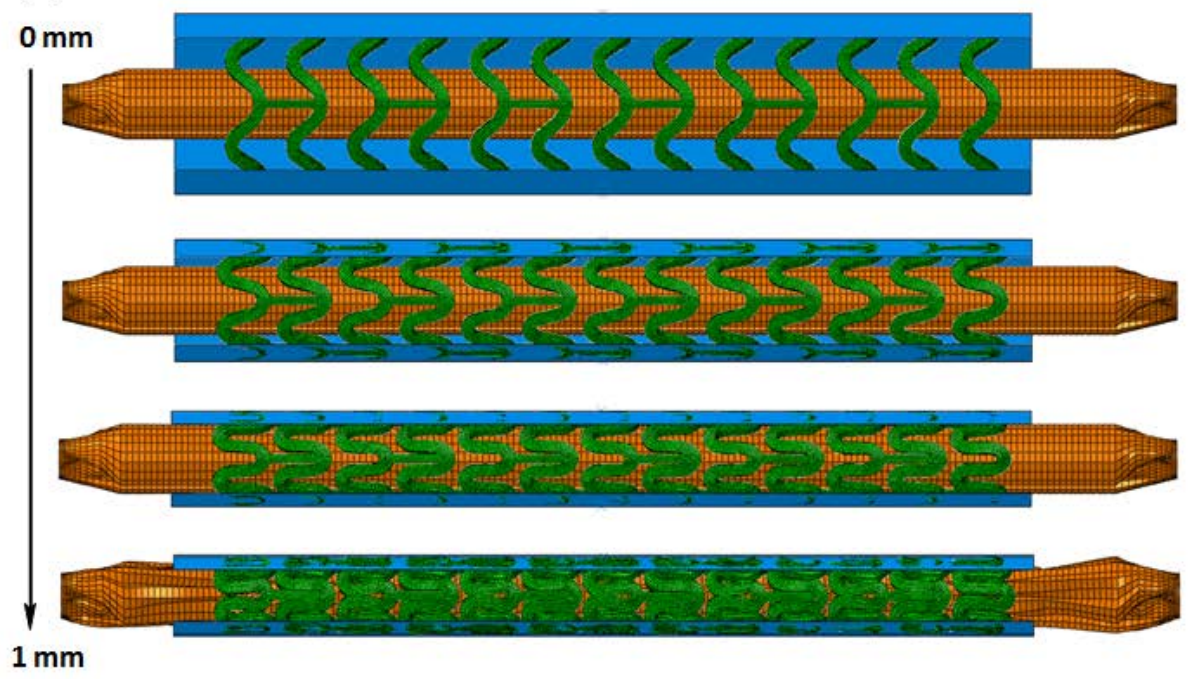

Fig. 4, FE mesh for balloon-scaffold assembly (a), assembly of 12 rigid plates (b), and simulation of Absorb scaffold crimping by applying $1 \mathrm{~mm}$ displacement loading on rigid plates (c). 


\section{Crimping and expansion of scaffold in diseased artery}

\subsection{Finite element model}

FE models for the Absorb scaffold and a tri-folded balloon are the same as described above. For the artery, a three-layered model was created, and had an inner diameter of $3 \mathrm{~mm}$ and a length of $40 \mathrm{~mm}$ (Fig. 5). The overall thickness of arterial wall is $1 \mathrm{~mm}$, including $0.38 \mathrm{~mm}$ adventitia layer, $0.33 \mathrm{~mm}$ media layer and $0.29 \mathrm{~mm}$ intima layer (Fig. 5). Here, the artery refers to left anterior descending coronary artery, with the thicknesses of individual layers given in Holzapfel et al. (2005). Plaque was modelled as a symmetric layer inside the artery, with a length of $10 \mathrm{~mm}$ and a stenosis of 50\% (inner diameter is $1.5 \mathrm{~mm}$ ). Hexahedral brick elements with reduced integration (C3D8R) behaviour were used to mesh the artery and plaque (Fig. 5). In the radial direction, the artery was meshed with 4 rows of elements for each tissue layer and the plaque was meshed with 8 rows of elements (Fig. 5). In the longitudinal direction, the element size for artery was increasing from the middle to both end sides by using bias control method (Fig. 5).

\subsection{Constitutive models for plaque-artery}

Constitutive models for Absorb scaffold and tri-folded balloon are the same as described above. For three-layered artery, the Holzapfel-Gasser-Ogden (HGO) model was used to describe the anisotropic behaviour of the arterial layers (with collagen fibres). In this study, it is assumed that there are two families of collagen fibres embedded in a soft incompressible matrix within each arterial layer. The model was calibrated by taking the average stress-stretch curves that were measured and obtained from 13 patients (Holzapfel et al., 2005). The calibrated parameter values for the HGO model were given in our previous paper (Schiavone et al., 2017) and the simulated longitudinal and circumferential stress-stretch responses of three layers were in excellent agreement with the experimental results (Schiavone et al., 2017). For the hypocellular plaque, the first order hyper elastic Ogden strain energy potential (Ogden, 1972) was used here, and material 
parameters were calibrated against test data (Loree et al., 1994) and given in Zahedmanesh and Lally (2009). Our own simulations verified that the stress-strain curve modelled with these parameters was in good agreement with experimental data reported in Loree et al. (1994).

\subsection{Simulation of crimping and deployment}

Similarly, in scaffold crimping process, 12 rigid plates were first assembled around the scaffold (Fig. 4b), and the distance between central axis of scaffold and rigid plate is $1.6 \mathrm{~mm}$. A linearly increasing displacement loading was applied on the surface of 12 rigid plates along radial direction, to squeeze the original expanded scaffold. The maximum displacement is $1 \mathrm{~mm}$. As a result, the biodegradable polymeric scaffold was radially compressed down to an outer diameter of $1.2 \mathrm{~mm}$. The scaffold was crimped onto the balloon in the end, meanwhile both ends of balloon were fully constrained because they were fixed on the catheter in the actual situation (Fig. 4c). After crimping, an additional step was added to remove the 12 rigid plates, allowing the crimped scaffold to spring back freely (Fig. 4c). Fig. 5 shows the scaffold-balloon-artery assembly after crimping process.

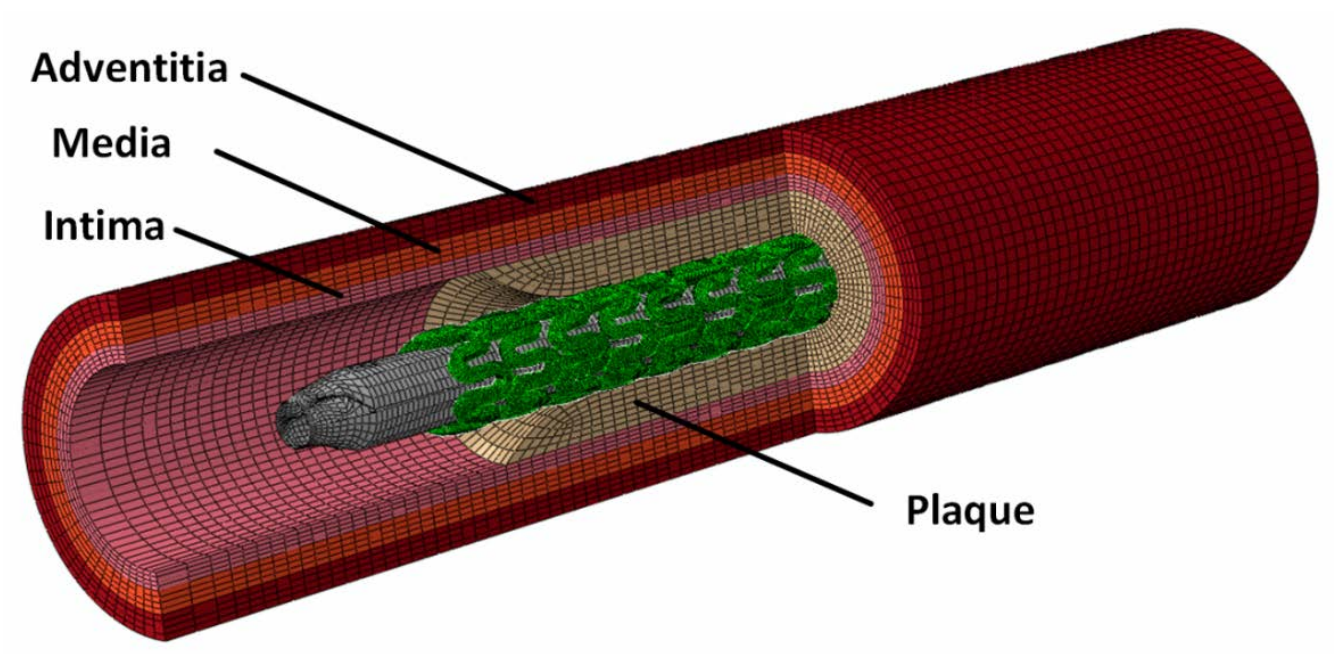

Fig. 5, Crimped scaffold-balloon-artery assembly obtained from crimping process. 
Scaffold expansion in diseased artery procedure consists of inflation step and deflation step. The inflation step was performed by applying pressure on the inner surface of balloon. The uniform pressure was linearly increasing from 0 to $1.4 \mathrm{MPa}$. Both ends of the artery were fully constrained to describe the constraint in the human body environment. The applied constraints are also at a significant distance from the edges of the scaffold and have minimal effects on the stress-strain distributions in the region of interest (i.e., diseased or stented region). Interactions between the artery, scaffold and balloon were modelled as surface to surface hard contacts with a frictional coefficient of 0.25 (Ju et al., 2008). Subsequently, the deflation step was modelled by releasing the pressure on the inner surface of balloon, which allowed the expanded scaffold to recoil freely. Interactions between scaffold, the balloon and the plaque were maintained in this step.

\section{Modelling of scaffold-artery interaction during degradation}

Fig. 6 shows the full simulation procedures to investigate the scaffold-artery interaction during degradation process. Firstly, pre-processing was performed to implant the scaffold in stenotic artery. In the pre-processing procedure, crimping, spring back (crimp recoil), inflation and deflation steps were simulated, by implementing the original PLLA stress-strain behaviour. The geometry and mesh for the scaffold and artery/plaque were described above. Subsequently, scaffold degradation process was modelled by changing the PLLA stress-strain behaviour as a function of degradation time. The change in PLLA stress-strain behaviour was controlled by a field variable, i.e., the mechanical property of polymer PLLA depends on the field variable values. Field variable was used to link stress-strain behaviour with degradation time. In this paper, different values (from 1 to 11) of field variable were used to represent the stress-strain behaviour at 11 degradation time points (i.e., day $0,30,92,189,272,365,442,545,601,659$ and 729) 
considered in this paper. The simulations over degradation were carried out in 11 consecutive steps, and for each step, a unique value of field variable is assigned, based on which the corresponding material behaviour is picked up automatically by ABAQUS for the simulations. At the same time, cyclic blood pressure was applied to capture the pulsatile vasomotion of artery. In order to simulate the systolic-diastolic fluctuations, a pressure in realistic waveform fluctuating between $80 \mathrm{mmHg}$ and $120 \mathrm{mmHg}$ (Fig. 7), was applied on the inner surface scaffold and artery, and 10 cycles were modelled in this study. The residual stresses induced to the scaffold by the deployment were not directly considered in our simulations. This is a limitation of the current study as the ABAQUS solver does not allow us to consider both residual stress and material property change simultaneously. But we believe that residual stresses will also change gradually and eventually diminish during material degradation. Future work is required to characterize how residual stresses will be reduced over degradation times. However, the in vitro degradation data (Fig. 2), and hence the calibrated stress-strain curves over degradation times, already accounted for material history effects such as any plastic strains, evolution of crystallinity and stress relaxation incurred during scaffold deployment as well as any effects due to residual stresses presented in the scaffolds at the beginning and changes thereof during degradation. In other words, any local or regional degradation interactions with scaffold history (crimping and expansion) and residual stresses are factored in the stress-strain curves calibrated and used in the finite element simulations (e.g. Fig. 10). 


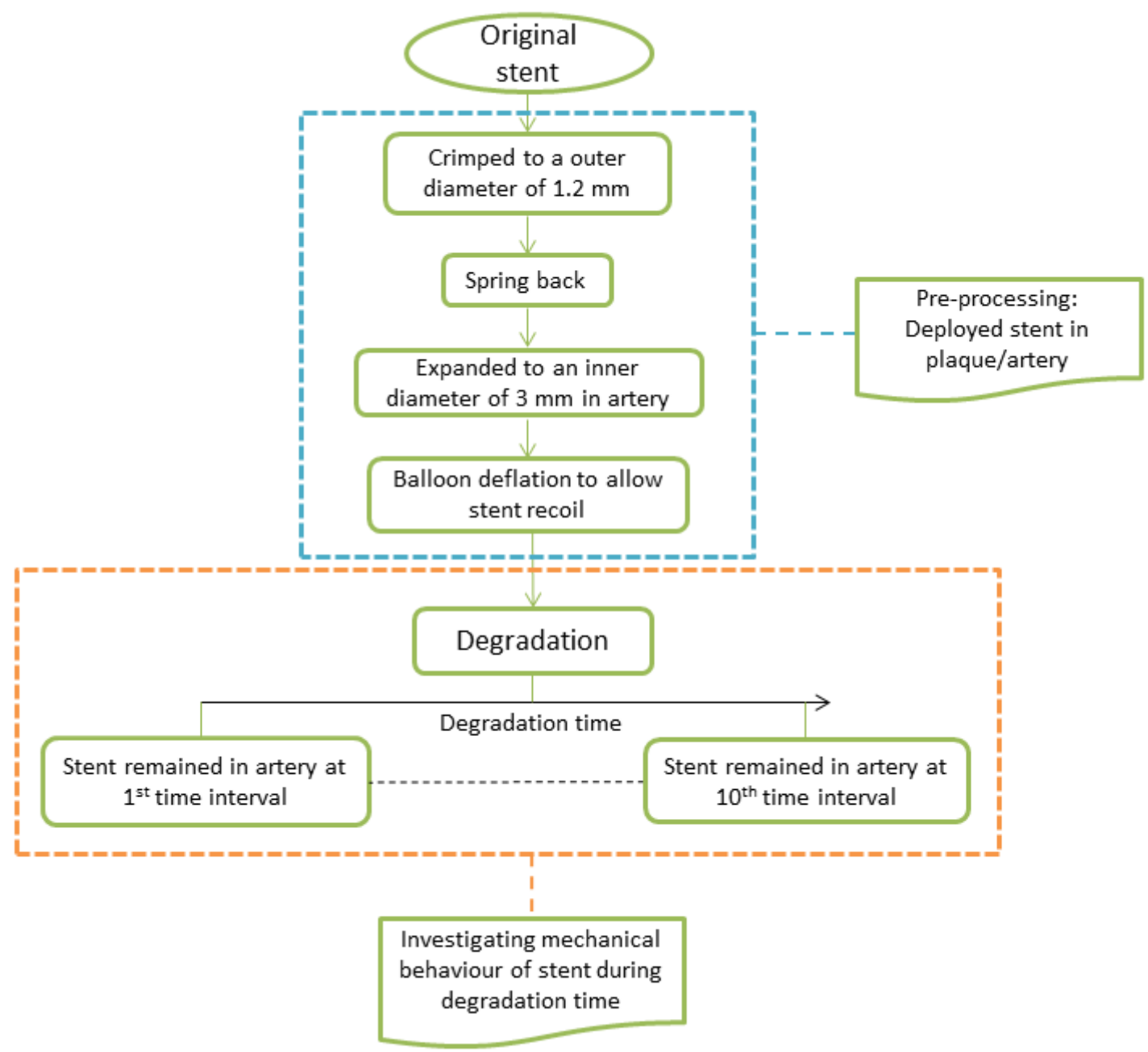

Fig. 6, Flow chart for the simulation procedure.

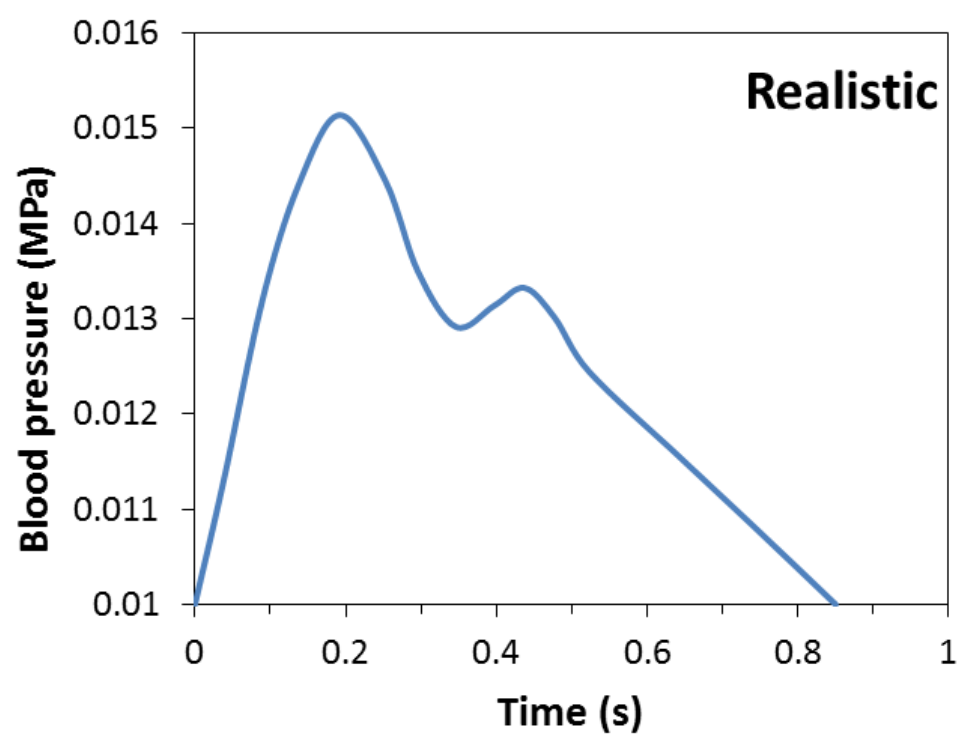

Fig. 7, Blood pressure with realistic waveforms. 


\section{Modelling of scaffold-artery interaction during vessel remodelling}

Generally, lumen area is measured by intravascular ultrasound method, which showed an increase over 2-year period of degradation for Absorb. This is referred as vessel remodelling. In this study, vessel remodelling was realised by changing the lumen diameter of artery-plaque system manually, based on the clinical data in literature. According to Serruys et al. (2013), the mean vessel area, mean lumen area and mean plaque area increased from $14.13 \mathrm{~mm}^{2}, 6.43 \mathrm{~mm}^{2}$ and $7.7 \mathrm{~mm}^{2}$ at post-procedure to $15.31 \mathrm{~mm}^{2}, 6.89 \mathrm{~mm}^{2}$ and $8.42 \mathrm{~mm}^{2}$ at 2 years for patients implanted with Absorb scaffolds. By translating to diameter change, it was found that the vessel outer diameter, the lumen diameter and plaque thickness were increased by $4.1 \%, 3.5 \%$ and $5.3 \%$ over two years. This percentage of increase has been used in this study to simulate the effect vessel remodelling on scaffold-artery interaction. Specifically, the plaque-artery configuration obtained directly from the simulation of scaffold deployment was taken as a reference state (day 0), which gave a vessel diameter of $5.38 \mathrm{~mm}$ ("D" in Fig. 8), a lumen diameter of $2.26 \mathrm{~mm}$ ("d" in Fig. 8) and a plaque thickness of $0.64 \mathrm{~mm}$ ("t" in Fig. 8). Over two years, the vessel diameter, lumen diameter and plaque thickness increased to $5.60 \mathrm{~mm}, 2.34 \mathrm{~mm}$ and $0.67 \mathrm{~mm}$ (see Fig. 8 and Table 1). For blood vessel, it was the thickening of media layer that contributed to the vessel area increase and consequently, only the thickness change of media layers (a $9.0 \%$ increase) was considered while the other two layers were kept unchanged. Then the manually constructed artery-plaque model, with the implanted scaffold inside, was used to simulate the scaffold-artery interaction after vessel remodelling over 2 years. Again, cyclic systolic-diastolic blood pressure (Fig. 7) was applied to the scaffold-artery system. It should be noted that the degraded stressstrain curve calibrated for 792 days was used for simulation of scaffold-artery interaction. For the reference state (i.e., 0 day or immediately after deployment), the original stress-strain curve was used for simulation of scaffold-artery interaction. 


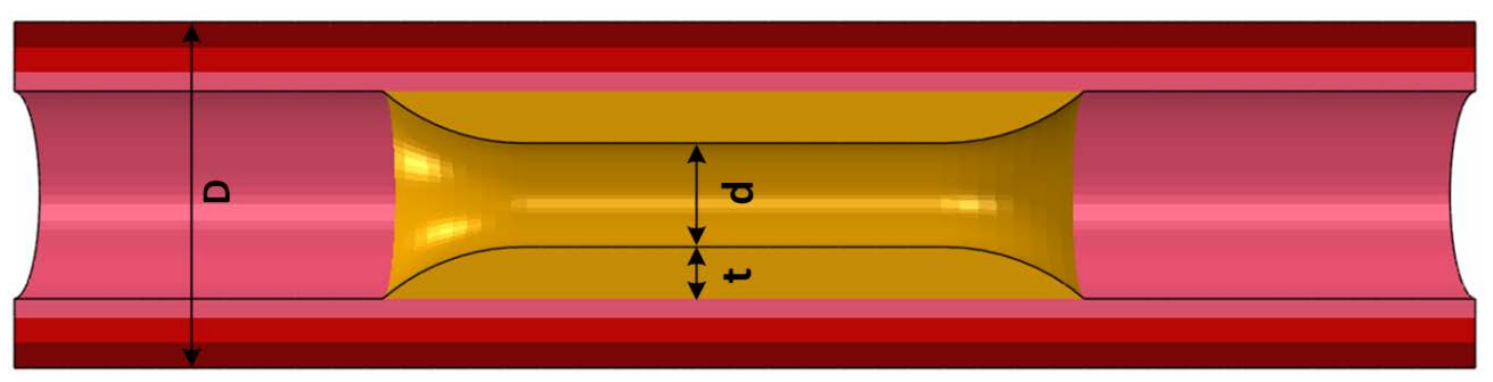

Fig. 8, Schematic of vessel and lumen diameters for artery-plaque system.

Table 1, Values of vessel diameter, lumen diameter and plaque thickness used in simulation.

\begin{tabular}{lcc}
\hline & $\begin{array}{c}\text { No remodelling } \\
(0 \text { day })\end{array}$ & $\begin{array}{c}\text { With remodelling } \\
(792 \text { days })\end{array}$ \\
\hline Vessel diameter D (mm) & 5.38 & 5.6 \\
Lumen diameter d (mm) & 2.26 & 2.34 \\
Plaque thickness t $(\mathrm{mm})$ & 0.64 & 0.67 \\
\hline
\end{tabular}

As part of changing the geometric boundaries of each artery layer (akin to "surface remodelling"), cross sectional thickness was increased for both plaque and media in our modelling approach to capture passive aspects of vessel remodelling. These geometric changes affect the remodelled plaque-artery stiffness and its chronic interaction with the scaffold, but do not address any changes of material properties occurring in the artery layer over time. The increase of media thickness applied in our models approximates the neointimal thickening behaviour that is known to occur in the intima-media layers after stenting and closely associated with the extent of vascular injury (Rogers and Edelman, 1995; Sullivan et al., 2002; Bennett, 2003). This was prescribed in a way that is compatible with the percentage change prescribed by Serruys et al. (2013). The exact delineation between the intima and media layers is difficult to discern in histologic practice so the increase prescribed in our work was applied to the media layer only. In addition, smooth muscle cells are known to infiltrate from the media into the intima during instent restenosis. This supports that the media effectively "grows" as we modelled it. However, 
we did not model any cellular changes in this work which is hugely complex and beyond the scope of current work.

\section{Results}

\subsection{PLLA stress-strain behaviour over degradation}

Fig. 9 shows the radial pressure-diameter curve obtained from simulation of re-crimping process for the Absorb scaffold, using the original PLLA stress-strain behaviour (i.e., no degradation). The dashed line followed the linear portion of the pressure-diameter curve, and the dasheddotted line is the $0.1 \mathrm{~mm}$ offset line. As a result, the calculated radial stiffness and strength are $4625 \mathrm{mmHg} / \mathrm{mm}(61.66 \mathrm{MPa})$ and $1900 \mathrm{mmHg}(25.33 \mathrm{MPa})$, respectively, for the Absorb scaffold at day 0 . Following the same method, the radial stiffness and strength values for the scaffold can be obtained using artificially constructed PLLA stress-strain curves at different degradation time points. The artificially constructed stress-strain curves were then tuned until the obtained radial stiffness and strength values, normalised against those at day 0 , matched the experimental measurements in Fig. 2. Here, it was assumed that the rate of plastic hardening was not affected by degradation. 


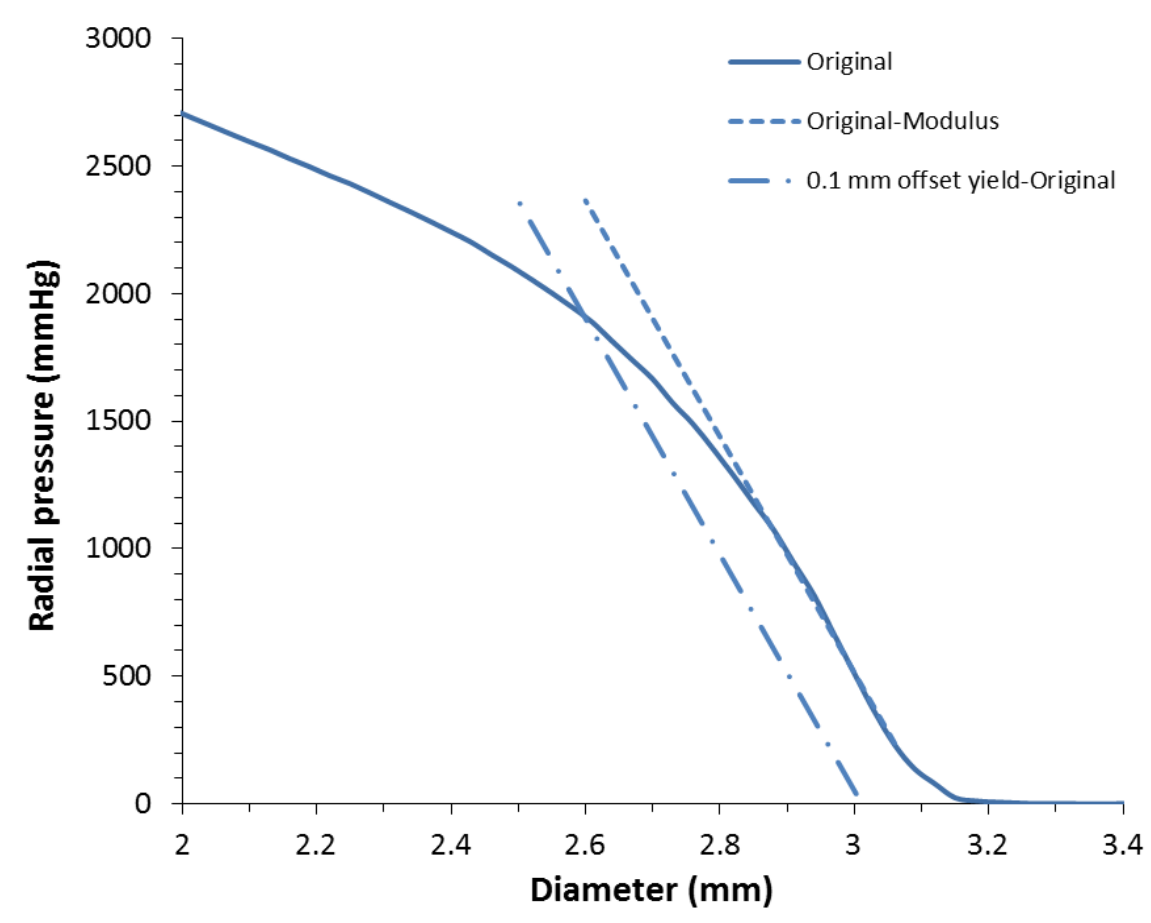

Fig. 9, Radial pressure-diameter curve obtained from re-crimping process for Absorb scaffold.

The back calculated PLLA stress-strain curves are given in Fig. 10 at different degradation time points. "Back calculation" refers to obtaining the stress-strain curve of the material which gives the same radial stiffness and strength of the scaffold as experimental measurements. This involves an iterative adjustment process until the simulated radial stiffness and strength match the experimental data (See Section 2.2 and Fig. 3). The normalised radial stiffness and strength for the scaffold, obtained using calibrated PLLA stress-strain curves at different degradation time points, are shown in Fig.11, which are in agreement with the experimental data. In Fig. 10, the red line with square symbols refers to the original stress-strain behaviour for PLLA. Overall, there is no significant change in the Young's modulus, because of small difference is observed for the radial stiffness of scaffold that is mainly controlled by Young's modulus of polymer. While the yield stress has a significant increase in the early time points (up to 365 days) due to the recrystallization of polymer, and then decreases quickly. 


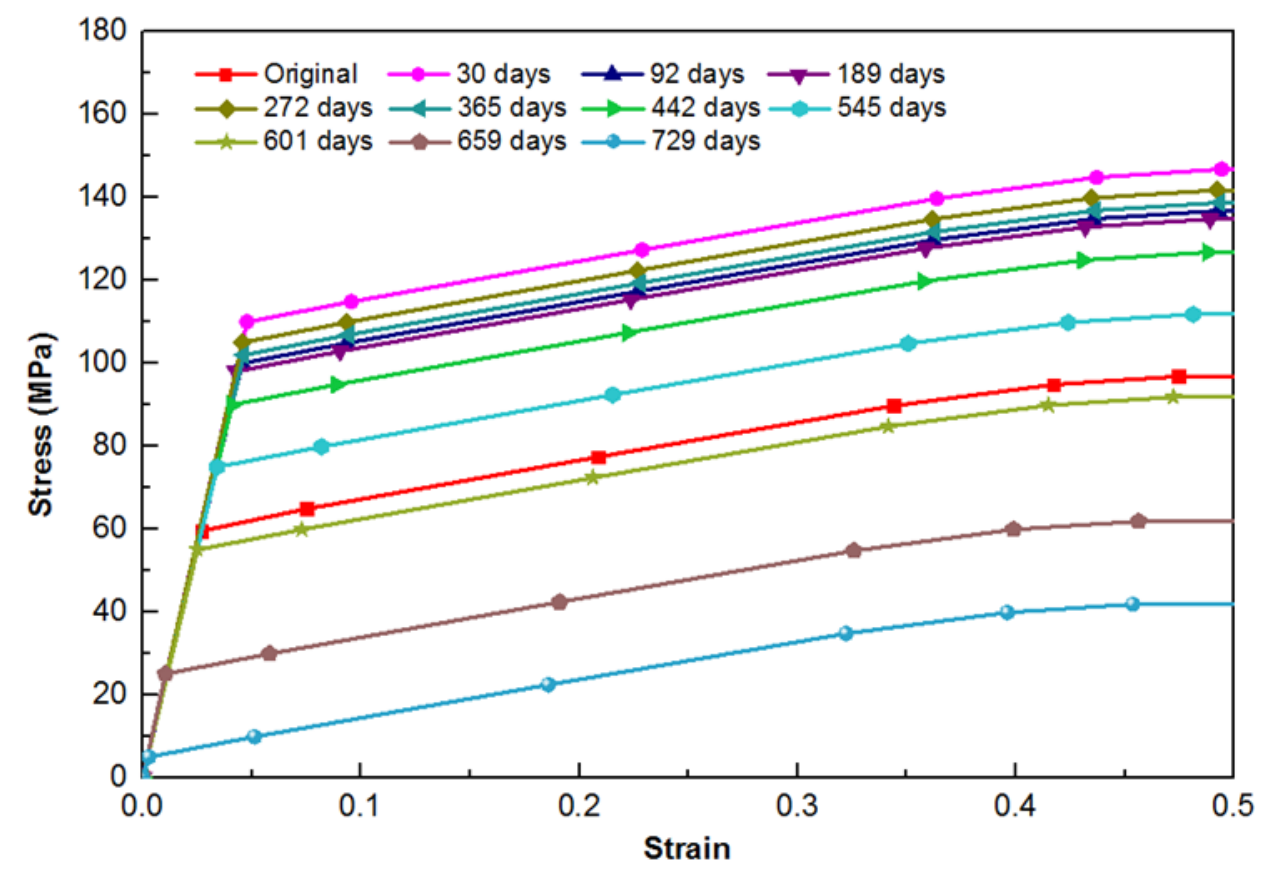

Fig. 10, Constructed PLLA stress-strain curves for Absorb scaffold at different degradation time points.

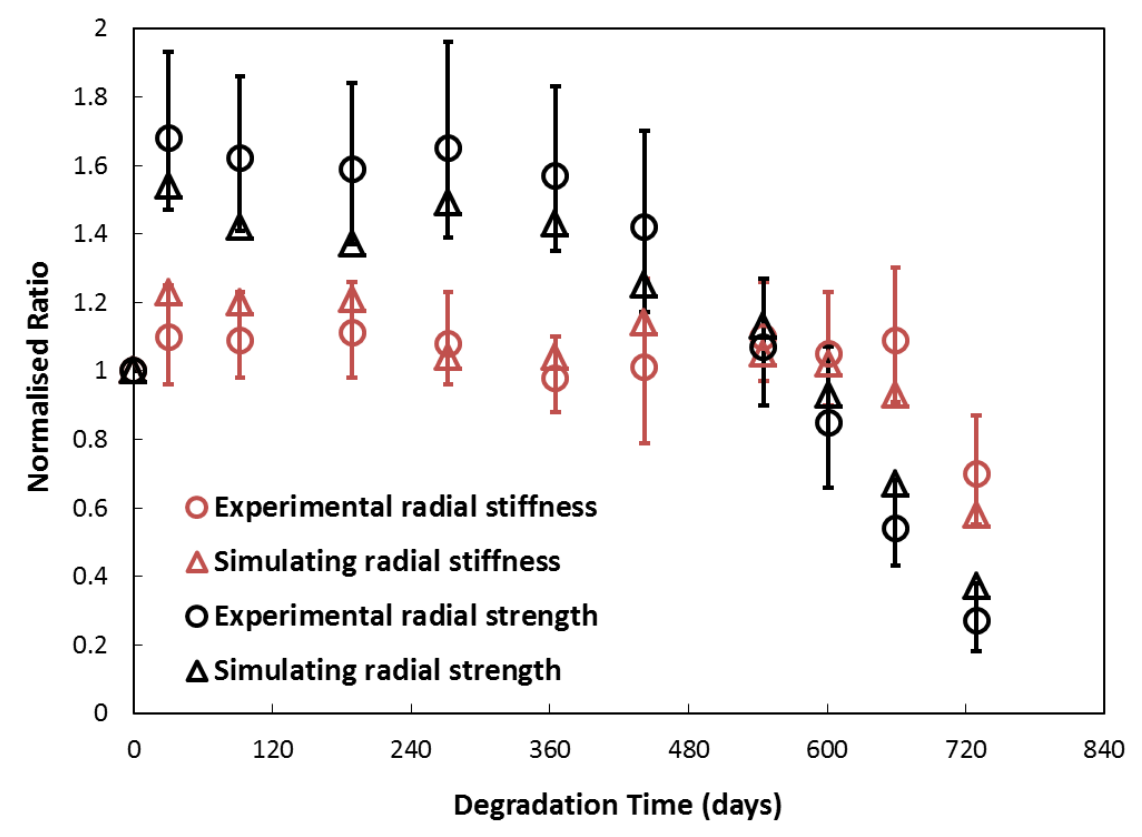

Fig. 11, Radial strength and stiffness data obtained from simulation for Absorb scaffold compared to experimental data at different degradation time points.

\subsection{Stress variation on the scaffold over degradation}

Degradation caused significant variations for the von Mises stress on the scaffold (Fig. 12).

Clearly, high stresses (red zones) are mainly located at the corners of U-bend struts, especially in 
the middle part of the scaffold structure. Due to the weakening of material property, stress concentration spreads towards both ends of scaffold, indicating a spatial change of stress distribution as a result of temporal degradation. The maximum von Mises stress value on the scaffold is $101.93 \mathrm{MPa}$ (Fig. 12a), 120.39 MPa (Fig. 12b), 116.68 MPa (Fig. 12c), 66.70 MPa (Fig. 12d) and $43.67 \mathrm{MPa}$ (Fig. 12e) at degradation time of 0 day, 189 days, 365 days, 659 days and 729 days, respectively.

Fig. 13 plots the variation of maximum von Mises stress on the scaffold over the degradation time of 729 days. It could be concluded that the maximum von Mises stress increases up to 365 days and then decreases quickly, corresponding to the change in stress-strain behaviour of scaffold material. Between 30 days and 365 days, no significant difference is observed for the maximum stress value on the scaffold, because the stress-strain behaviour of scaffold material is very similar over the period. Beyond 365 days, the stress starts to reduce, with dramatic reduction after 545 days. Specifically, the maximum von Mises stress is reduced from 115.16 $\mathrm{MPa}$ at 545 days to $43.67 \mathrm{MPa}$ at 729 days (Fig. 13). Significant stress loss indicates that the biodegradable scaffold might lose mechanical integrity after 545 days when it is likely being absorbed by the human body. In this section, we did not consider the effect of vessel remodelling, so plaque and artery experienced no change. Vessel remodelling (i.e., geometrical change) is considered in Section 6.4. 
von Mises stress (MPa)

(a)
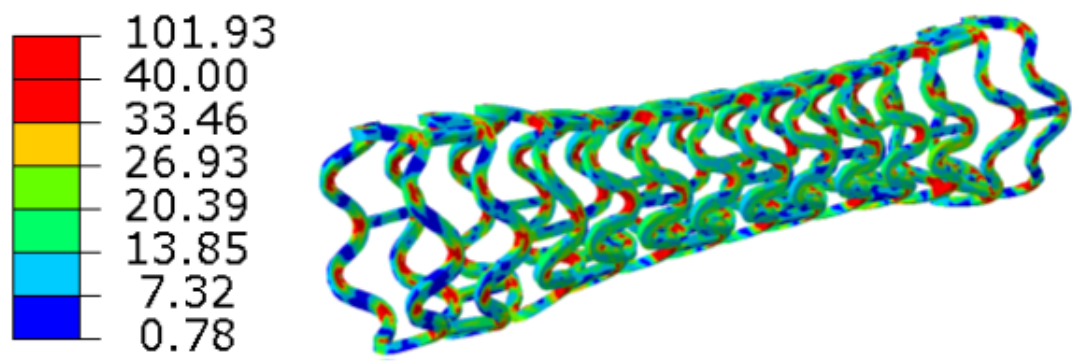

(b)
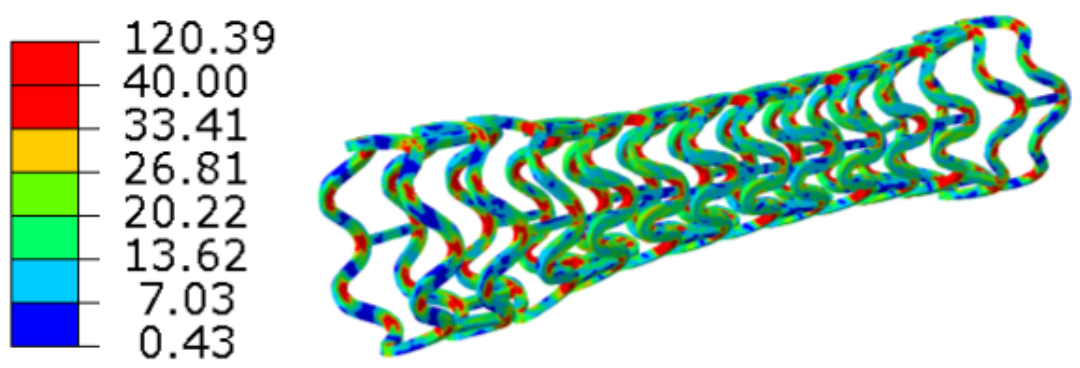

(c)
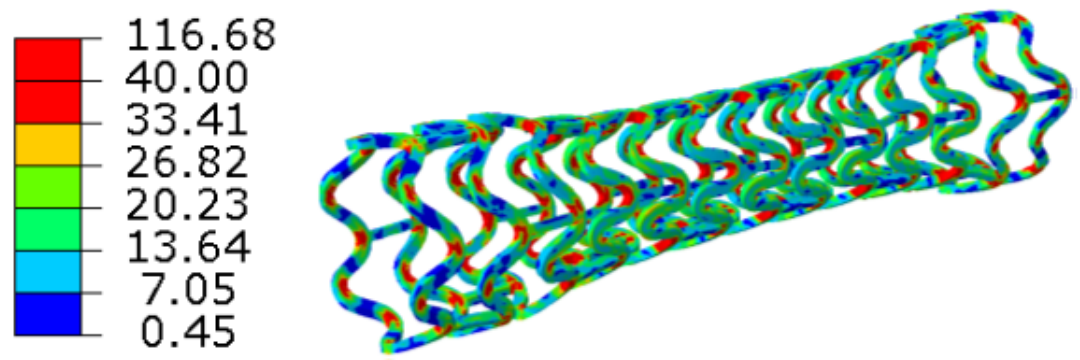

(d)
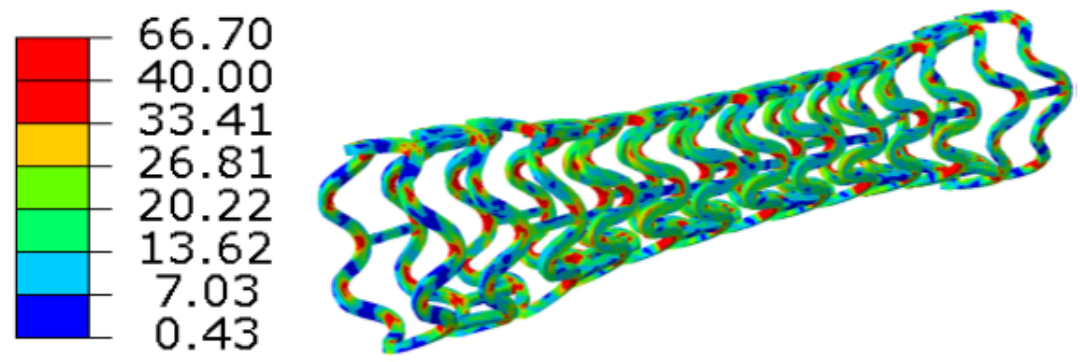

(e)
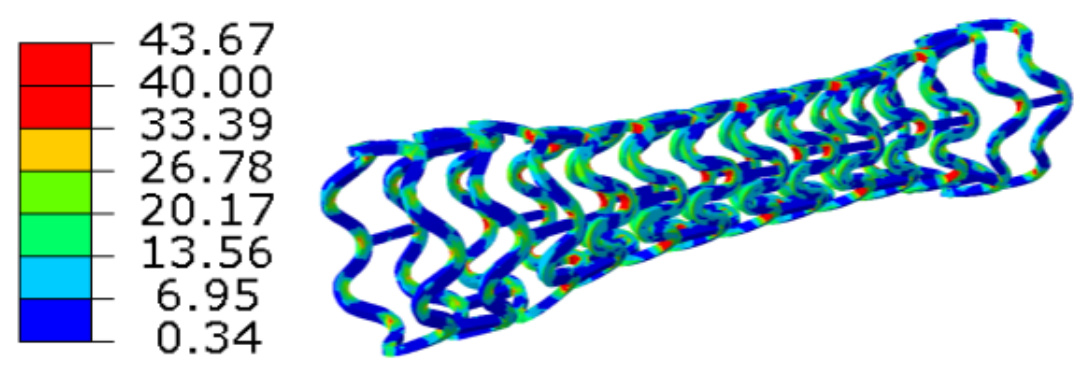

Fig. 12, Von Mises stress distribution on the scaffold at a degradation time of 0 day (a), 189 days

(b), 365 days (c), 659 days (d) and 729 days (e). 


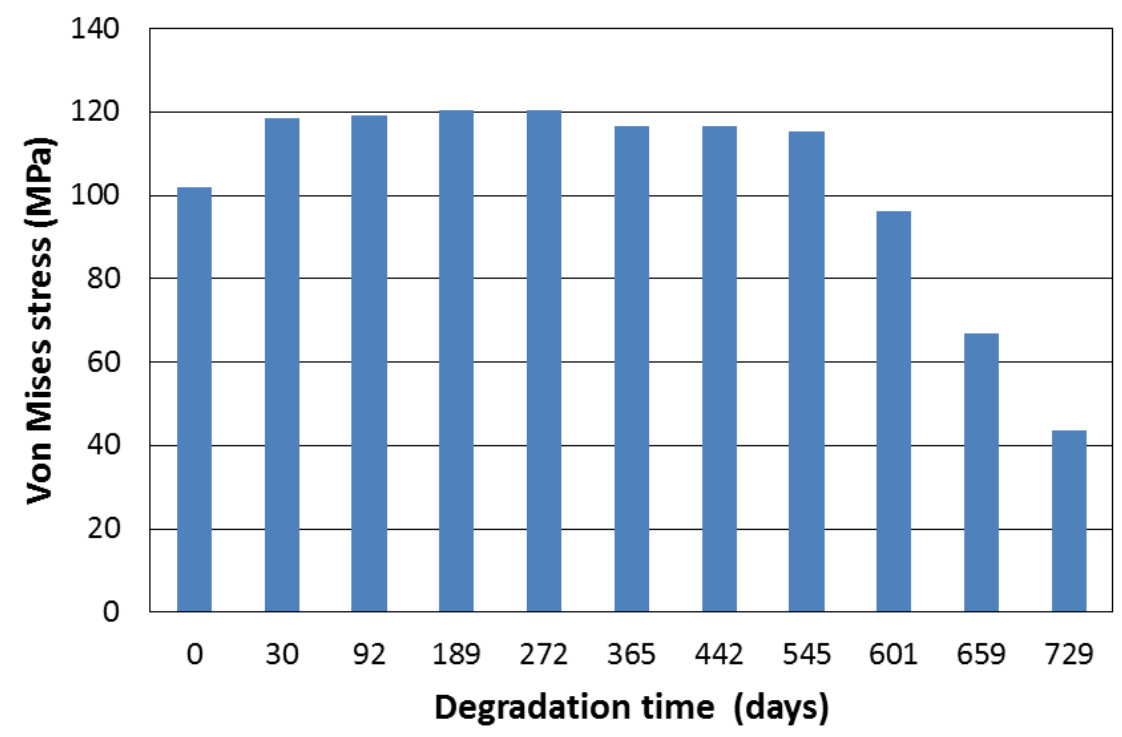

Fig. 13, Maximum von Mises stress on the scaffold over degradation.

\subsection{Stress variation in the artery-plaque system over degradation}

From the contour plot of the maximum principal stress on the plaque (Fig. 14), it is seen that the peak value continuously decreases with degradation after six months of implantation, with values of $0.711 \mathrm{MPa}$ (Fig. 14b), $0.702 \mathrm{MPa}$ (Fig. 14c), $0.662 \mathrm{MPa}$ (Fig. 14d) and $0.500 \mathrm{MPa}$ (Fig. 14e) at degradation times of 189 days, 365 days, 659 days and 729 days, respectively. In addition, the area exhibiting higher stresses (red zone) also decreases progressively during scaffold degradation. As a result, the regions of high-level stress shrink significantly at both ends of plaque where a strong contact exists between the scaffold and plaque due to the dogboning effect (Fig. 14). The lower stress, combined with a smaller region of stress concentration, is an indication of reduced mechanical load on the vessel wall. Stress reduction is a gradual process and according to our simulations, significant arterial stress reduction occurs after one and half years of degradation. This behaviour is expected since the scaffold is designed to disappear over two years (i.e., when no longer needed to support the artery) and arterial stress reduction in this later time period occurs after the scaffold struts have been integrated with overgrown tissue (Serruys et al., 2013). 
This means that degrading scaffold struts are unlikely to directly interact with long-term blood flow.

(a)
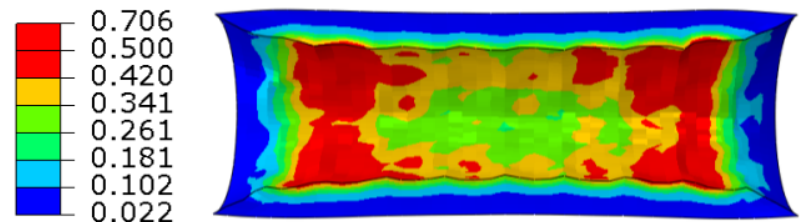

(b)
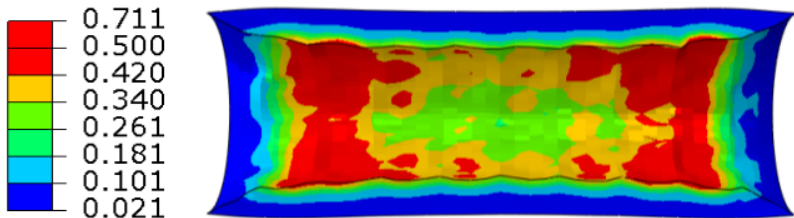

(c)
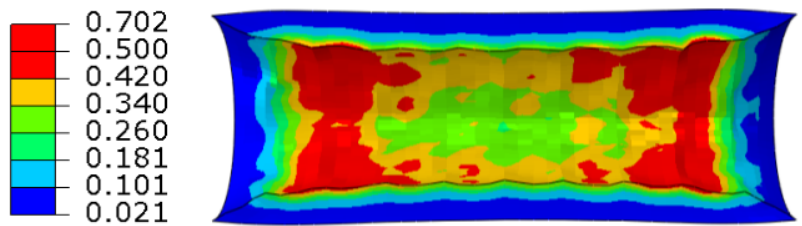

(d)
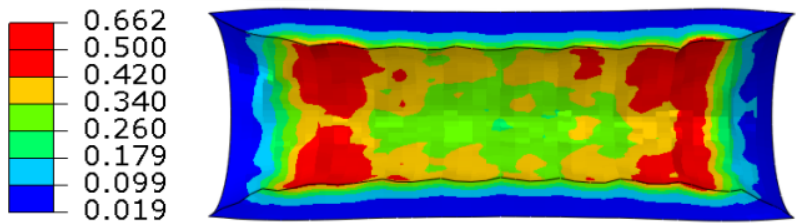

(e)
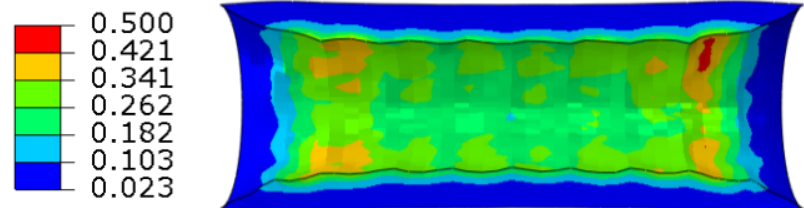

Fig. 14, Contour plot of the maximum principal stress on plaque at a degradation time of 0 day

$$
\text { (a), } 189 \text { days (b), } 365 \text { days (c), } 659 \text { days (d) and } 729 \text { days (e). }
$$

The contour plots of the maximum principal stress in the intima, media and adventitia layers are shown in Fig. 15 to Fig. 17, respectively. For the intima layer, the peak maximum principal stress increases significantly for the segment covered with plaque (i.e., from $0.266 \mathrm{MPa}$ at day 0 to $0.281 \mathrm{MPa}$ at day 189; Fig. 15a and b), followed by a gradual decrease over the rest period of degradation (i.e., $0.276 \mathrm{MPa}$ at 365 days, $0.254 \mathrm{MPa}$ at 659 days and $0.192 \mathrm{MPa}$ at 729 days; Fig. $15 \mathrm{c}, \mathrm{d}$ and e). The stress in the intima layer is concentrated over the plaque-covered segment, 
especially at both end sides due to scaffold dogboning (Fig. 15). For the media layer, a gradual decrease was found for the peak maximum principal stress over degradation time, with a value of 0.022 $\mathrm{MPa}$ at day zero (Fig. 16a) and a reduction to $0.021 \mathrm{MPa}$ (4.5\% reduction; Fig. 16b), 0.019 $\mathrm{MPa}(13.6 \%$ reduction; Fig. 16c), $0.017 \mathrm{MPa}(22.7 \%$ reduction; Fig. $16 \mathrm{~d})$ and $0.013 \mathrm{MPa}(40.9 \%$ reduction; Fig. 16e) at day 189, 365, 659 and 729, respectively. The area of high level stress is also considerably reduced (Fig. 16). For the adventitia layer, both the peak maximum principal stress and the stress-concentration area are similar at a degradation time of up to 659 days (Fig. 17). Specifically, the peak maximum principal stress has a magnitude of 0.01-0.009 $\mathrm{MPa}$, and the stress concentration is again found at both ends of plaque-covered segment (Fig. 17). After 729 days, a clear reduction of stress concentration area is observed while the peak maximum principal stress remains almost unchanged (0.008 MPa; Fig. 17e).

(a)
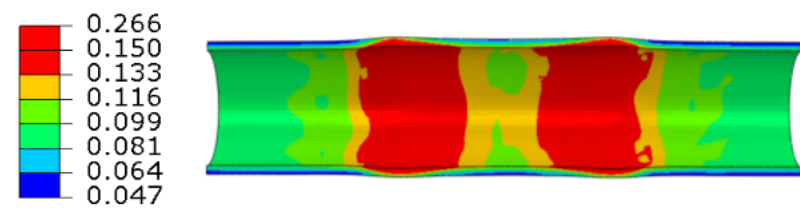

(b)
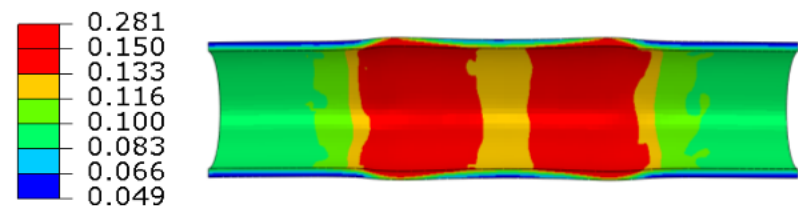

(c)
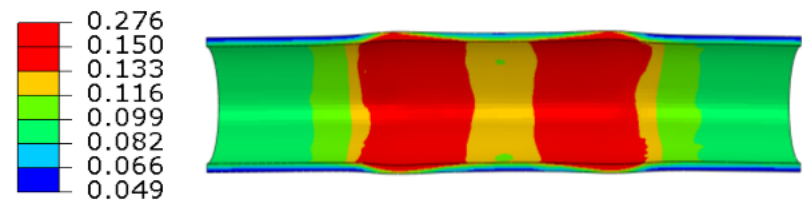

(d)
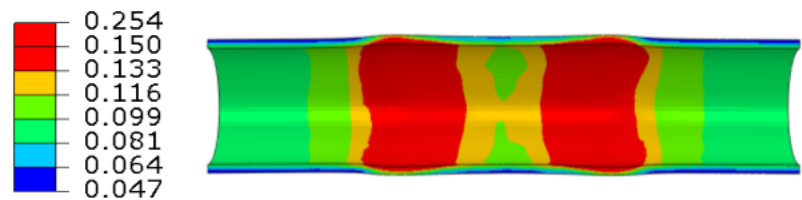

(e)
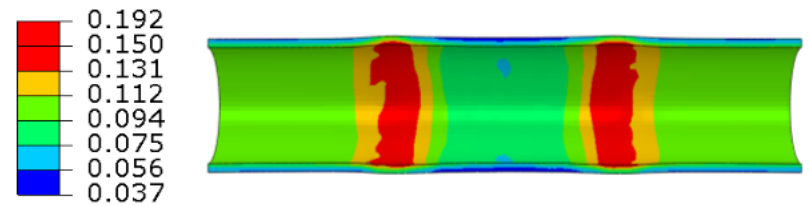

Fig. 15, Contour plot of the maximum principal stress on the intima layer at a degradation time of 0 day (a), 189 days (b), 365 days (c), 659 days (d) and 729 days (e). 
(a)

$\square \begin{aligned} & 0.022 \\ & 0.012 \\ & 0.010 \\ & 0.009 \\ & 0.007\end{aligned}$

0.007

0.005

0.004
0.002

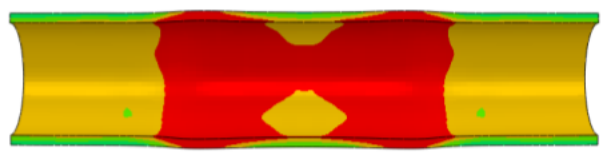

0.021
-0.012
0.010

(b)

0.010

0.009

0.007
0.005

0.003

0.002

0.017

0.012
0.010
0.008

(c)
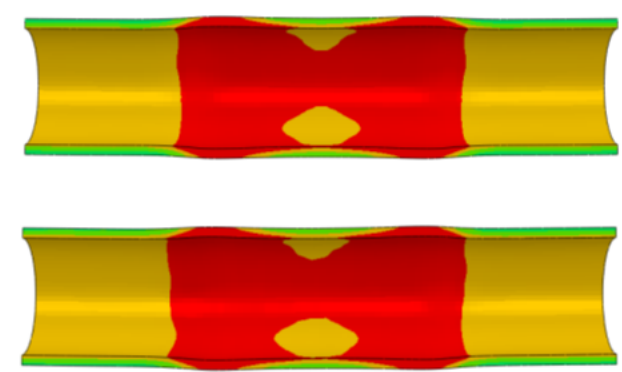

(d)
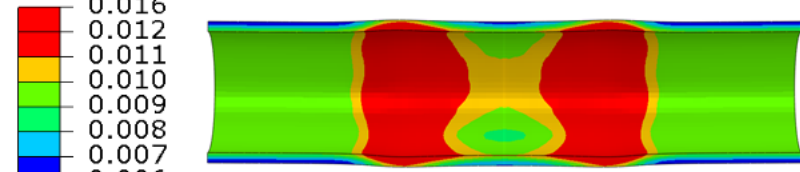

0.013

0.012

(e)
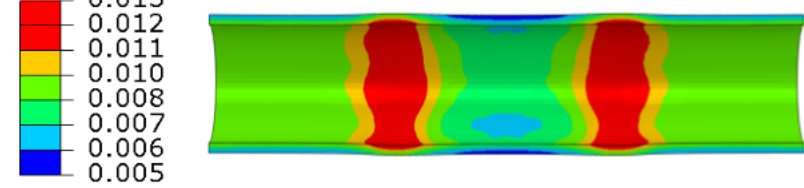

Fig. 16, Contour plot of the maximum principal stress on the media layer at a degradation time of 0 day (a), 189 days (b), 365 days (c), 659 days (d) and 729 days (e). 
(a)
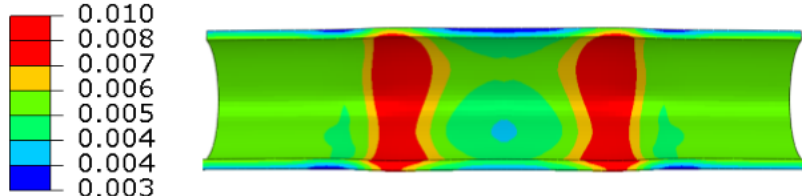

(b)
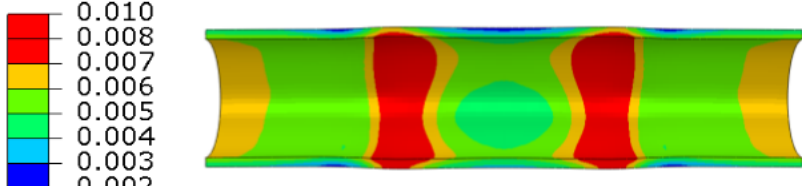

(c)

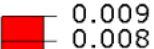

0.008

c)

0.007

$-0.006$

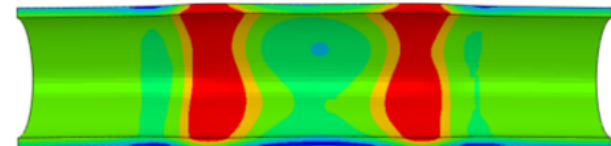

)
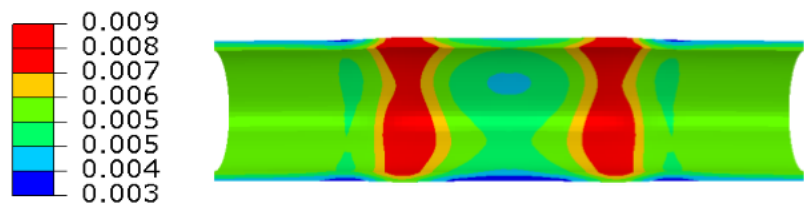

(e)
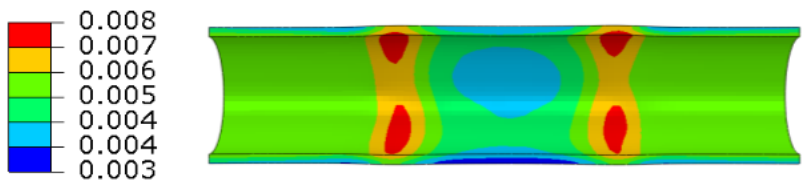

Fig. 17, Contour plot of the maximum principal stress on the adventitia layer at degradation time of 0 day (a), 189 days (b), 365 days (c), 659 days (d) and 729 days (e).

To provide further insights into stress variation in the artery over degradation, Fig. 18 shows the spatial distribution of the average maximum principal stress (volume average along the circumferential direction) for the three individual arterial layers along the longitudinal direction at degradation time of 0 day, 365 days and 729 days. Significant stress reduction is observed over the plaque-covered part of three individual arterial layers, especially from 365 days to 729 days of degradation time (Fig. 18). It could be concluded that the maximum principal stress on the plaque-artery system has a continuous reduction over degradation process after one-year implantation, due to the weakening of stress-strain behaviour of scaffold material. Stress reduction may in fact avoid inducing undesired chronic biological responses like cellular injury or inflammation, promoting vessel remodelling. 


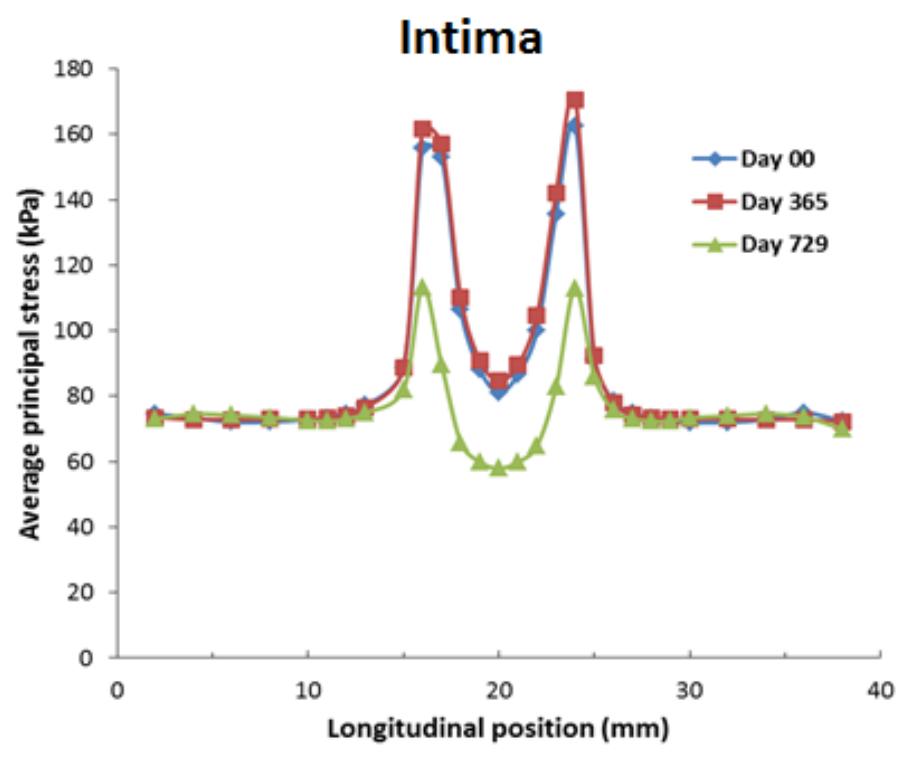

\section{Media}

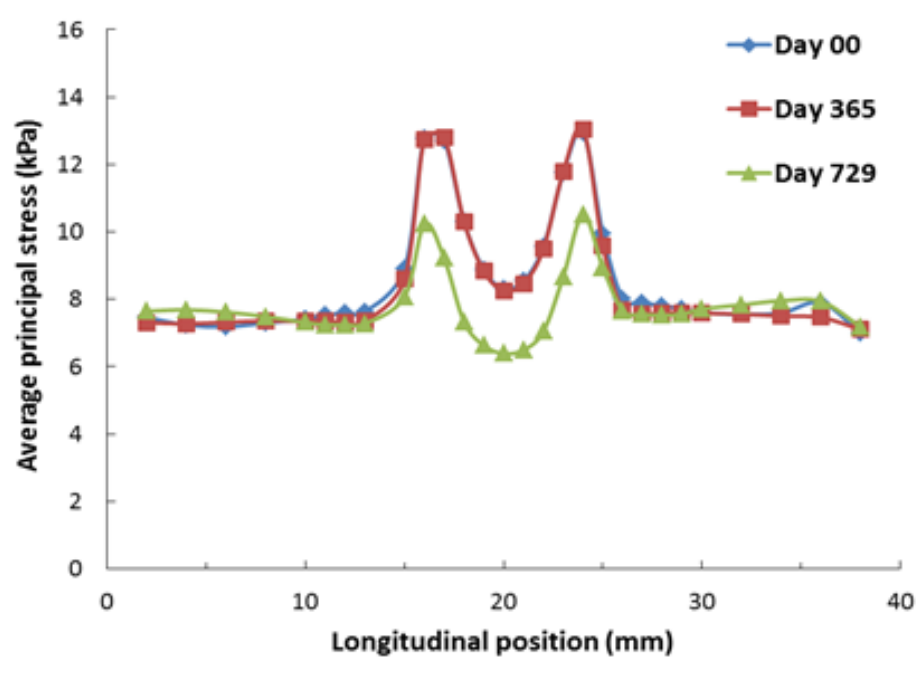

Adventitia

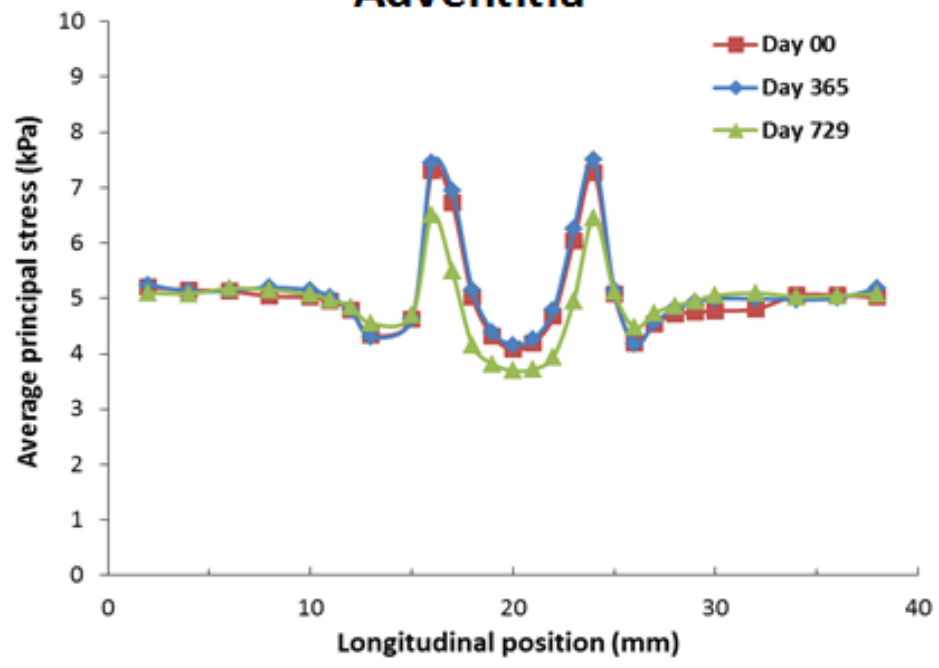

Fig. 18, The spatial distribution of the average maximum principal stress in three arterial layers at degradation time of 0 day, 365 days and 729 days. 


\subsection{Effects of vessel remodelling}

Contour plot of von Mises stress on the scaffold shows that stress distribution and reduction at two-year degradation time are similar to those presented above, indicating negligible effect of vessel remodelling. Basically, stresses are concentrated at the U-bend struts and significant stress reduction is observed on the scaffold over 2-year degradation time due to the loss of modulus and strength. It should be noted that residual stresses generated from scaffold crimping are not considered due to model limitations. Here, the stresses and deformation are mainly caused by the balloon inflation and the applied blood pressure.

\section{(a)}

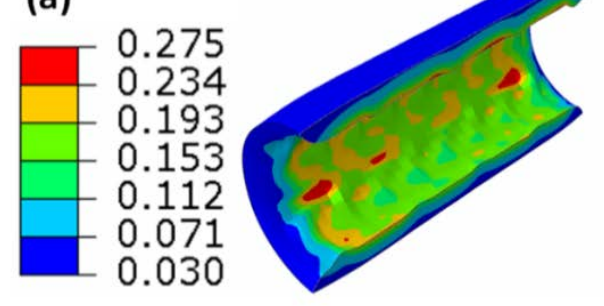

(b)

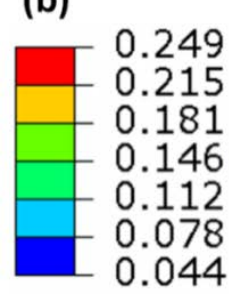

(c)

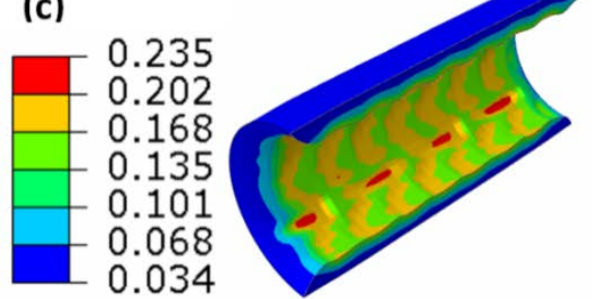

Fig. 19, The contour plot of von Mises stress on the plaque at 0 day (a) and 729 days without vessel remodelling (b) and with vessel remodelling (c) after 10 cycles of pulsatile blood pressure.

Fig. 19 shows the contour plot of von Mises stress on the plaque at 0 day and 729 days subjected to 10 cycles of pulsatile blood pressure. Stress concentrations are located at bridge strut-covered part of the plaque, and stress on the inner surface is higher than that on the outer surface. Also, stress reduction is observed on the plaque, which is around $0.040 \mathrm{MPa}(14.5 \%$; Fig.19c) and $0.026 \mathrm{MPa}$ (9.5\%; Fig.19b) for simulations with and without considering the vessel remodelling. 
This indicates that vessel remodelling is accompanied by further reduction of stresses in the scaffold-plaque system.

(a)

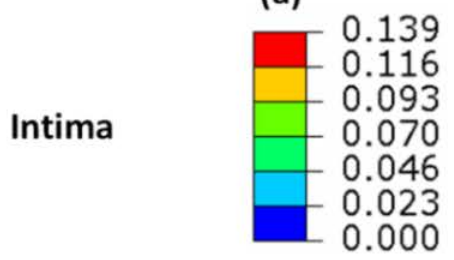

(b)
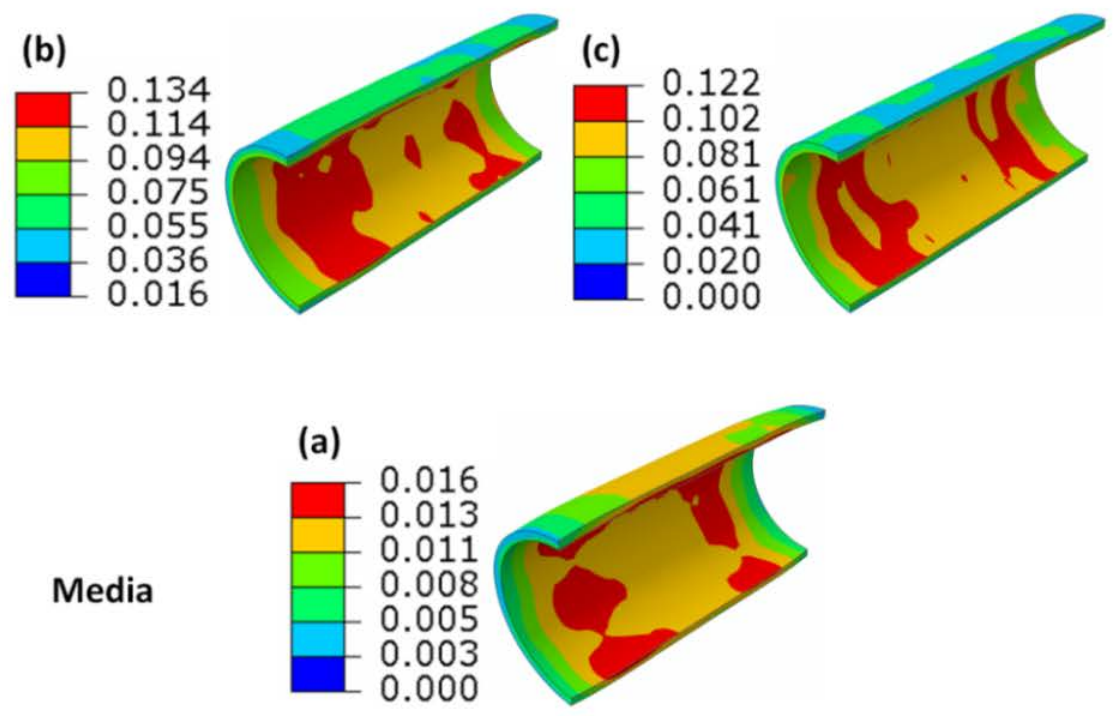

(b)
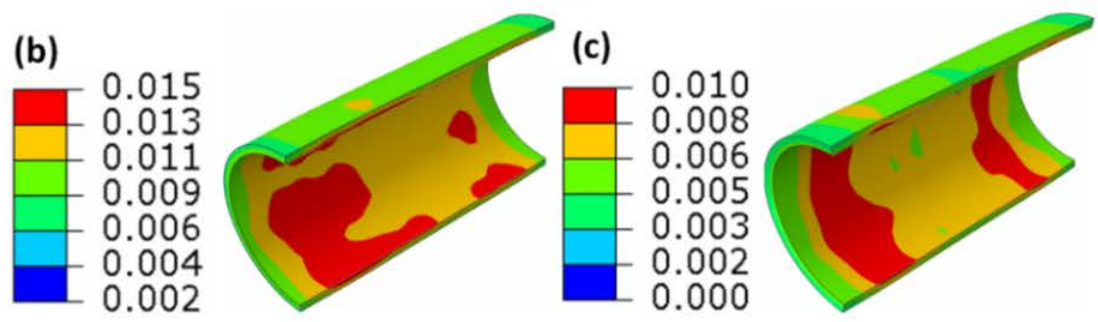

Adventitia

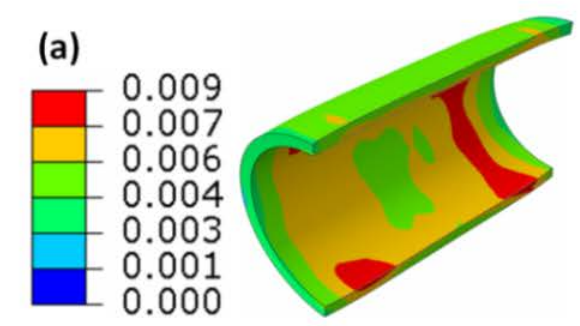

(b)
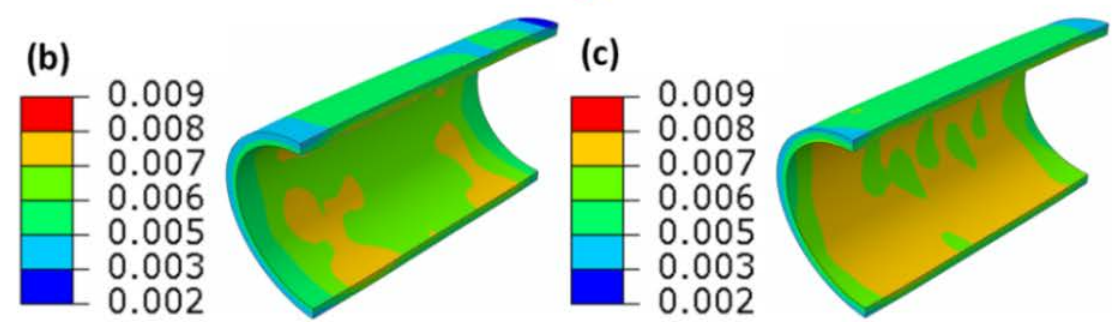
Fig. 20, The contour plot of von Mises stress on the intima, media and adventitia layers at 0 day (a) and 729 days without vessel remodelling (b) and with vessel remodelling (c) after 10 cycles of pulsatile blood pressure.

Contour plots of von Mises stress are also given for the three individual arterial layers at 0 day and 729 days (with and without vessel remodelling) after 10 cycles of pulsatile blood pressure (Fig. 20). Stresses are mainly concentrated at both ends of arterial layers, corresponding to the regions of stress concentration at the scaffold edges. For the intima layer, the maximum stress values are reduced by $0.017 \mathrm{MPa}(12.2 \%)$ and $0.005 \mathrm{MPa}(3.6 \%)$ for simulations with and without vessel remodelling (top subfigure in Fig. 20). For the media layer, relative stress loss is more significant, around 37.5\% (0.006 MPa) and 6.3\% (0.001 MPa) for simulations with and without vessel remodelling (middle subfigure in Fig. 20). For adventitia layers, it seems that no difference is observed for the maximum von Mises stress (bottom subfigure in Fig. 20). Vessel remodelling is based on follow up studies of clinical trials (Serruys, 2009; Serruys, 2010; Serruys, 2011 and Serruys, 2016). In terms of lumen gain, the observed vessel changes represent a positive remodelling process. However, the vessel cross-sectional area increased during remodelling. Due to the complex interplay of the biological processes which drives these remodelling changes, we mainly focused on effect of vessel remodelling (i.e., geometrical change) on the interaction between scaffold and blood vessel.

\section{Discussions}

Due to their biodegradation characteristics, bioresorbable scaffolds have advantages in overcoming long-term clinical complications (e.g. restenosis, thrombosis and inflammation) associated with permanent metallic stents. Degradation is also the key factor that influences the mechanical performance of bioresorbable scaffolds after deployment. However, existing studies have been focused on predicting the degradation behaviour of polymeric scaffolds, including the 
influence of deformation. For instance, Soares et al. (2010) applied a thermodynamically consistent constitutive model to predict degradation behaviour of PLLA scaffolds. Degradation of three different scaffold designs was modelled, and results showed that high risk of degradation was mainly observed at the bends of scaffold rings and junction points due to high levels of deformation. Luo et al. (2014) developed a numerical model to study the degradation behaviour of bioabsorbable PLLA scaffold, by combining the degree of degradation with prestretched deformation. Results showed that degradation happened throughout the whole scaffold over 30 days, but the degradation rate was not uniform. The maximum degradation was observed at the inner surface of U-bend strut where the stress concentration/maximum strain occurred during scaffold expansion, which was in good agreement with experimental result. Basically, this indicates that there is an interaction between deformation and degradation, and high levels of deformation or stress facilitate the transport of water into the material and accelerate the degradation process (Luo et al., 2104). However, there is little research on modelling the deformation of biodegradable polymeric scaffold by considering the effect of scaffold material degradation because of the lack of stress-strain data during degradation. This paper is the first attempt to obtain the mechanical response of PLLA during two-year degradation times. From the constructed stress-strain behaviour of PLLA, we can see that both the yield stress and modulus had an increase up to a period of 15 months, corresponding to the increase of radial strength and radial stiffness of PLLA scaffold determined in experiments. It indicates that the radial strength and stiffness of scaffold are mainly controlled by the yield stress and Young's modulus of scaffold material. From the material point of view, the initial increase of yield stress and Young's modulus might be caused by the recrystallization of biodegradable polymer PLLA due to the water absorption and temperature increase (from room temperature $20^{\circ} \mathrm{C}$ to human body temperature $37^{\circ} \mathrm{C}$ ). It was reported that crystallization can significantly improve the mechanical strength and stiffness of PLLA in literature (Perego, 1996). For instance, the amorphous PLLA presents a tensile strength of 55-59 MPa whereas the crystalline PLLA has 
a tensile strength of 60-70 $\mathrm{MPa}$. In addition, it was also reported that PLLA could be potential material candidate for applications at a temperature of $40^{\circ} \mathrm{C}$ or higher, because the crystallization can significantly improve the mechanical properties. In addition, the radial strength of scaffold reduced quickly after 365 days, but the significant reduction in radial stiffness occurred after 659 days. This suggested that the yield stress of PLLA was more sensitive to hydrolysis degradation compared to Young's modulus, i.e., degradation leads to faster change of yield stress compared to Young's modulus. This has not been reported in literature yet. However, this conclusion is based purely on our finite element simulation results, and requires supportive experimental evidence. Also, in this paper, we have not modelled the physical degradation of scaffold itself. We focused on the mechanical property change caused by degradation. Such behaviour is reflected by the stress plot of the scaffolds over two-year degradation times (see Fig. 12).

In percutaneous coronary intervention (PCI), scaffold implantation induces high stresses on the arterial wall, which may generate negative effects such as injury to arterial tissue. Therefore, it is important to evaluate the stress state generated in the plaque-artery system during scaffold deployment process. It is a challenge to measure the stresses generated in the scaffold-artery system using experimental methods, due to the complicated process and complex geometrical factors. So far, finite element method was dominantly used to reveal the stress state in the artery following the deployment of scaffolds. An ideal process of scaffold deployment requires an expansion which is sufficient to clear vessel obstructions and also induces minimal damage to the vessel walls (Farooq et al., 2011), as in-stent restenosis can be triggered by unsuccessful treatments including vessel over-stretch and injury, incomplete expansion of the scaffold and fracture of the scaffold struts. Both Imani et al. (2014) and Lally et al. (2005) confirmed a direct correlation between arterial wall stresses and restenosis rate. Similarly, study on scaffold-artery interaction during degradation is necessary to evaluate the safety and efficacy of polymeric 
scaffolds, but little work is available in the literature. Again, this paper is the first attempt to evaluate the mechanical interaction between a bioresorbable scaffold and a diseased artery over the process of scaffold degradation. According to our results, at the end of two-year degradation, a trend of stress reduction was generally observed for both scaffold and plaque-artery system in the simulation of scaffold-artery interaction by changing the stress-strain behaviour of scaffold material and applying pulsatile blood pressure. Over degradation times, the stress variation on the scaffold showed an increase firstly before a gradual decrease, which was consistent with the change of yield stress for the scaffold material. For scaffold, the maximum von Mises stress reduced to $43 \mathrm{MPa}$ at 729 days ( 2 years), indicating the loss of mechanical integrity and support due to scaffold degradation. As reported in a clinical follow-up study by Serruys et al. (2009), the bioresorbable everolimus-eluting scaffold was fully absorbed at 2 years as revealed by means of multi-imaging approaches including echogenicity, virtual history and optical CT. However, the stress on the plaque and arterial layers showed a general decrease over scaffold degradation. For the plaque-artery system, relatively high principal stress (0.706 MPa at 0 day) was generated when the scaffold was implanted through full expansion. Then, the principal stress on the plaqueartery system became lower and lower (reduced to $0.5 \mathrm{MPa}$ at 729 days) over degradation time when subjected to pulsatile blood pressure, which can be regarded as occurring in concert with the natural healing process of the artery.

Long-term lumen gain after implantation of Absorb scaffolds has been reported in a series of clinical trial studies (Serruys, 2009; Serruys, 2010; Serruys, 2011 and Serruys, 2016). The intravascular ultrasound examination showed that a positive increase occurred to the mean luminal area (as well as minimal luminal area) between 6 months and 2 years. These measurements provide evidence of vessel remodelling, or more specifically positive remodeling. Accompanying this lumen gain, an increase in plaque size and vessel area was also noticed. Vessel remodelling has been simulated in this study in order to reveal its effect on the stress- 
strain interaction between scaffolds and artery after two years of implantation. During vessel remodelling, both scaffold and plaque exhibited stress reduction. Stress reduction was also observed for the intima and media layers of artery, but almost no difference was found for the adventitia layer. This may offer an understanding of the healing process of injured vessel walls after scaffold deployment, from a mechanical point of view. Generally, vessel remodelling refers to a change of both geometry and mechanical property, caused by histological structure change and external loads. However, in this study, vessel remodelling was modelled by changing vessel geometry (increase the lumen diameter, plaque size and media layer thickness) manually, without considering the evolution of tissue property and its intrinsic residual stress. Also to our best knowledge, vessel remodelling is a very complex process and still not fully understood yet (Goel et al., 2012). The major biomechanical stimuli include contributions from both mechanical deformation and vessel haemodynamics such as wall stress, wall shear stress, blood pressure, blood flow and hypertension (Wentzel et al., 2001; Goel et al., 2012). For instance, low wall shear stress is associated with constrictive vascular remodelling while high wall stress is attributed to an increase in wall area (Wentzel et al., 2001). In addition, biological factors such as transforming growth factor beta (TGF- $\beta$ ) and matrix metalloproteinases (MMP) also play key roles in vessel remodelling after stenting (Goel et al., 2012). For instance, TGF- $\beta 1$ is a potent contributor to constrictive remodelling while MMP-9 overexpression was found to reduce lumen loss by promoting adaptive remodelling (Goel et al., 2012). But, it should be noted that it is very difficult to tell exactly how these factors govern vessel remodelling, due to the complexity of the topic as well as the sophisticated interactions between all the biomechanical and biological factors. Further work is required to elucidate such effects in detail, however, the present work offers a signal of how the artery-plaque system's mechanical behaviour generally changes over time.

Systolic-diastolic stress will introduce cyclic stresses within the scaffold. However, estimated fatigue stresses are considerably lower than the stresses imposed in the scaffold during acute 
expansion of the scaffold (stress amplitude less than 5\% of acute maximum stress during expansion; Qiu, 2017). Rigorous calculation of fatigue safety factors has been attempted for scaffolds in prior works (Li et al., 2010; Azaouzi et al., 2012), however, traditional Goodman analyses (mean stress effect) provide only a rough assessment of fatigue resistance suitable for simple comparisons between scaffold designs, given that this technique is best suited for predicting failure in ductile metals and not polymers. Due to the complexity of accurately predicting the fatigue behaviour of polymeric scaffolds, it is considered beyond the scope of this work and the capability of current modelling techniques. Experimental fatigue-resistance characterization (S-N curves) and long-term device verifications performed by manufacturers such as Abbott are not available to us due to confidentiality reasons, however, extensive clinical trial data for the Absorb scaffold have confirmed that the product is efficacious and promotes safe tissue coverage without any mentioned indications of scaffold fatigue fractures.

Finally, degradation behaviour of polymeric scaffolds was attempted by in vitro and in vivo methods, physical properties (e.g. mass weight and molecular weight) and mechanical properties (e.g., tensile strength and elastic modulus) showed a difference during scaffold degradation. A large number of clinical trials in human indicated the safety and efficacy of polymeric scaffolds in treatment of coronary artery disease, and a series of in vivo studies in animals also showed that the polymeric scaffolds offered the benefits for the treatment of biliary disease. However, experimental studies are still limited due to the microscale geometry of scaffold and complex environment of human artery, and systematic efforts are urgently required in order to provide an improved understanding of this both clinically and scientifically important phenomenon.

\section{Conclusions}

Scaffold-artery interaction was simulated by incorporating changes in scaffold material behaviour over time while simultaneously including vessel remodelling changes (such as lumen gain and 
plaque/artery thickening) that occur during the timeframe of scaffold degradation. The von Mises stress variation on the scaffold was consistent with the change of yield stress for scaffold material, whereas the stresses in the plaque-artery system generally reduced over time but varied by tissue layer. Overall, the results generated support that the interaction of the Absorb scaffold with the artery wall is stable throughout the first year after deployment, and is thereafter associated with gradually reducing stresses in the plaque-artery system as the vessel remodels. It seems that property degradation is mainly responsible for stress drop in the system, with additional contributions from vessel remodelling. Also, this work represents a first attempt at studying scaffold-vessel interaction by combining material property degradation behaviour with anticipated vessel wall changes after deployment of a bioresorbable scaffold. As such, modelling limitations are present but can be overcome in future work.

\section{Competing interests}

The authors declare that they have no competing interests

\section{Acknowledgement}

The work was financially supported by Abbott Vascular, 3200 Lakeside Drive, Santa Clara, CA 95054, USA.

\section{References}

Azaouzi, M., Makradi, A., Belouettar, S., 2012. Fatigue life prediction of cardiovascular stent using finite element method. Computer Methods in Biomechanics and Biomedical Engineering 15, 93-95.

Bennett M.R., 2003. In-stent stenosis: pathology and implications for the development of drug eluting stents. Heart 89, 218-224. 
Chen, Y., Zhou, S., Li, Q., 2011. Mathematical modeling of degradation for bulk-erosive polymers: Applications in tissue engineering scaffolds and drug delivery systems. Acta Biomaterialia 7, 1140-1149.

Farooq, V., Gogas, B.D., Serruys, P.W., 2011. Restenosis delineating the numerous causes of drug-eluting stent restenosis. Circulation: Cardiovascular Interventions 4 (2), 195-205.

Goel, S.A., Guo, L.-W., Liu, B., Kent, K.C., 2012. Mechanisms of post-intervention arterial remodelling. Cardiovascular Research 96, 363-371.

Grabow, N., Bünger, C.M., Sternberg, K., Mews, S., Schmohl, K., Schmitz, K.P., 2007. Mechanical properties of a biodegradable balloon-expandable stent from poly (L-lactide) for peripheral vascular applications. Journal of Medical Devices 1(1), 84-88.

Gervaso, F., Capelli, C., Petrini, L., Lattanzio, S., Di Virgilio, L., Migliavacca, F., 2008. On the effects of different strategies in modelling balloon-expandable stenting by means of finite element method. Journal of Biomechanics 41(6), 1206-1212.

Gong, Y., Zhou, Q., Gao, C., Shen, J., 2007. In vitro and in vivo degradability and cytocompatibility of poly (1-lactic acid) scaffold fabricated by a gelatin particle leaching method. Acta Biomaterialia 3(4), 531-540.

Holzapfel, G.A., Sommer, G., Gasser, C.T., Regitnig, P., 2005. Determination of layer-specific mechanical properties of human coronary arteries with nonatherosclerotic intimal thickening and related constitutive modeling. American Journal of Physiology-Heart and Circulatory Physiology 289(5), H2048-H2058.

Imani, S.M., Goudarzi, A., Ghasemi, S., Kalani, A., Mahdinejad, J., 2014. Analysis of the Stent Expansion in a Stenosed Artery Using Finite Element Method: Application to Stent Versus Stent Study. Proceedings of the Institution of Mechanical Engineers, Part H: Journal of Engineering in Medicine 228(10), 996-1004.

Ju, F., Xia, Z., Sasaki, K., 2008. On the finite element modelling of balloon-expandable stents. Journal of the Mechanical Behavior of Biomedical Materials 1(1), 86-95. 
Lally, C., Dolan, F., Prendergast, P. 2005. Cardiovascular Stent Design and Vessel Stresses: A Finite Element Analysis. Journal of Biomechanics 38(8), 1574-1581.

Lane, J.P., Perkins, L.E., Sheehy, A.J., Pacheco, E.J., Frie, M.P., Lambert, B.J., Rapoza, R.J., Virmani, R., 2014. Lumen gain and restoration of pulsatility after implantation of a bioresorbable vascular scaffold in porcine coronary arteries. JACC: Cardiovascular Interventions 7(6), 688-695

Li, J., Luo, Q., Xie, Z., Li, Y., Zeng, Y., 2010. Fatigue life analysis and experimental verification of coronary stent. Heart and Vessels 25, 333-337.

Liu, G., Zhang, X., Wang, D., 2014. Tailoring Crystallization: Towards High-Performance Poly (lactic acid). Advanced Materials 26(40), 6905-6911.

Loree, H.M., Grodzinsky, A.J., Park, S.Y., Gibson, L.J., Lee, R.T., 1994. Static circumferential tangential modulus of human atherosclerotic tissue. Journal of Biomechanics 27(2), 195-204.

Luo, Q., Liu, X., Li, Z., Huang, C., Zhang, W., Meng, J., Chang, Z., Hua, Z., 2014. Degradation model of bioabsorbable cardiovascular stents. PloS one 9(11), e110278.

Lyu, S., Untereker, D., 2009. Degradability of polymers for implantable biomedical devices. International Journal of Molecular Sciences 10(9), 4033-4065.

Engineer, C., Parikh, J., Raval, A., 2011. Review on hydrolytic degradation behavior of biodegradable polymers from controlled drug delivery system. Trends in Biomaterials \& Artificial Organs 25(2), 79-85.

Pauck, R.G., Reddy, B.D., 2015. Computational analysis of the radial mechanical performance of PLLA coronary artery stents.. Medical Engineering \& Physics 1(37), 7-12.

Ogden, R., 1972. Large deformation isotropic elasticity-on the correlation of theory and experiment for incompressible rubberlike solids. In Proceedings of the Royal Society of London A: Mathematical, Physical and Engineering Sciences 326(1567), 565-584.

Ormiston, J.A., Serruys, P.W., Onuma, Y., van Geuns, R.J., de Bruyne, B., Dudek, D., Thuesen, L., Smits, P.C., Chevalier, B., McClean, D., Koolen, J., 2012. First serial assessment at 6 
months and 2 years of the second generation of absorb everolimus-eluting bioresorbable vascular scaffold. Circulation: Cardiovascular Interventions 5(5), 620-632.

Ormiston, J.A., Serruys, P.W., Regar, E., Dudek, D., Thuesen, L., Webster, M.W., Onuma, Y., Garcia-Garcia, H.M., McGreevy, R., Veldhof, S., 2008. A bioabsorbable everolimus-eluting coronary stent system for patients with single de-novo coronary artery lesions (ABSORB): a prospective open-label trial. The Lancet 371(9616), 899-907.

Perego, G., Cella, G.D., Bastioli, C., 1996. Effect of molecular weight and crystallinity on poly (lactic acid) mechanical properties. Journal of Applied Polymer Science 59(1), 37-43.

Prabhu, S., Hossainy, S., 2006. Modeling of degradation and drug release from a biodegradable stent coating. Journal of Biomedical Materials Research Part A 80A, 732-741.

Qiu, T.Y., 2017. A computational study of mechanical behaviour of bioresorbable polymeric stents. PhD thesis, Loughborough University, UK.

Rodrigues, N., Benning, M., Ferreira, A.M., Dixon, L., Dalgarno, K., 2016. Manufacture and Characterisation of Porous PLA Scaffolds. Procedia CIRP 49, 33-38.

Rogers, C., Edelman, E.R., 1995. Endovascular stent design dictates experimental restenosis and thrombosis. Circulation 91, 2995-3001.

Schiavone, A., Abunassar, C., Hossainy, S., Zhao, L.G., 2016. Computational analysis of mechanical stress-strain interaction of a bioresorbable scaffold with blood vessel. Journal of Biomechanics 49(13), 2677-2683.

Schiavone, A., Qiu, T. and Zhao, L.G., 2017. Crimping and deployment of metallic and polymeric stents-finite element modelling. Vessel Plus 2017, 1 (1), 12-21.

Serruys, P.W., Onuma, Y., Dudek, D., Smits, P.C., Koolen, J., Chevalier, B., de Bruyne, B., Thuesen, L., McClean, D., van Geuns, R.J., Windecker, S., 2011. Evaluation of the second generation of a bioresorbable everolimus-eluting vascular scaffold for the treatment of de novo coronary artery stenosis: 12-month clinical and imaging outcomes. Journal of the American College of Cardiology 58(15), 1578-1588. 
Serruys, P.W., Onuma, Y., Ormiston, J.A., de Bruyne, B., Regar, E., Dudek, D., Thuesen, L., Smits, P.C., Chevalier, B., McClean, D., Koolen, J., 2010. Evaluation of the Second Generation of a Bioresorbable Everolimus Drug-Eluting Vascular Scaffold for Treatment of De Novo Coronary Artery StenosisClinical Perspective. Circulation 122(22), 2301-2312.

Serruys, P.W., Ormiston, J.A., Onuma, Y., Regar, E., Gonzalo, N., Garcia-Garcia, H.M., Nieman, K., Bruining, N., Dorange, C., Miquel-Hébert, K., Veldhof, S., 2013. A bioabsorbable everolimus-eluting coronary stent system (ABSORB): 2-year outcomes and results from multiple imaging methods. The Lancet 373(9667), 897-910.

Serruys, P.W., Ormiston, J., van Geuns, R.J., de Bruyne, B., Dudek, D., Christiansen, E., Chevalier, B., Smits, P., McClean, D., Koolen, J., Windecker, S., 2016. A polylactide bioresorbable scaffold eluting everolimus for treatment of coronary stenosis. Journal of the American College of Cardiology 67(7), 766-776.

Soares, J.S., Moore Jr, J.E., Rajagopal, K.R., 2008. Constitutive framework for biodegradable polymers with applications to biodegradable stents. Asaio Journal 54(3), 295-301.

Soares, J.S., Moore, J.E., Rajagopal, K.R., 2010. Modeling of deformation-accelerated breakdown of polylactic acid biodegradable stents. Journal of Medical Devices 4(4), 041007.

Sullivan, T.M., Ainsworth, S.D., Langan, E.M., Taylor, S., Snyder, B., Cull, D., Youkey, J., Laberge, M., 2002. Effect of endovascular stent strut geometry on vascular injury, myointimal hyperplasia, and restenosis. Journal of V ascular Surgery 36, 143-149

Vieira, A.C., Vieira, J.C., Ferra, J.M., Magalhães, F.D., Guedes, R.M., Marques, A.T., 2011. Mechanical study of PLA-PCL fibers during in vitro degradation. Journal of the Mechanical Behavior of Biomedical Materials 4(3), 451-460.

Wentzel, J.J., Kloet, J., Andhyiswara, I., Oomen, J.A.F., Schuurbiers, J.C.H., de Smet, B.J.G.L., Post, M.J., de Kleijn, D., Pasterkamp, G., Borst, C., Slager, C.J., Krams, R., 2001. Shearstress and wall-stress regulation of vascular remodeling after balloon angioplasty effect of matrix metalloproteinase inhibition. Circulation 104, 91-96. 
Zahedmanesh, H., Lally, C., 2009. Determination of the influence of stent strut thickness using the finite element method: implications for vascular injury and in-stent restenosis. Medical \& Biological Engineering \& Computing 47(4), 385-393.

Zamiri, P., Kuang, Y., Sharma, U., Ng, T.F., Busold, R.H., Rago, A.P., Core, L.A., Palasis, M., 2010. The biocompatibility of rapidly degrading polymeric stents in porcine carotid arteries. Biomaterials 31(31), 7847-7855.

Zhu, X., Braatz, R.D., 2014. Modeling and analysis of drug-eluting stents with biodegradable PLGA coating: consequences on intravascular drug delivery. Journal of Biomechanical Engineering - Transactions of the ASME 136, 111004. 